\title{
WestVirginiaUniversity
}

THE RESEARCH REPOSITORY @ WVU

Graduate Theses, Dissertations, and Problem Reports

2014

\section{A preliminary study evaluating potential probiotic use in endodontics}

Khaled Seifelnasr

West Virginia University

Follow this and additional works at: https://researchrepository.wvu.edu/etd

\section{Recommended Citation}

Seifelnasr, Khaled, "A preliminary study evaluating potential probiotic use in endodontics" (2014). Graduate Theses, Dissertations, and Problem Reports. 588.

https://researchrepository.wvu.edu/etd/588

This Thesis is protected by copyright and/or related rights. It has been brought to you by the The Research Repository @ WVU with permission from the rights-holder(s). You are free to use this Thesis in any way that is permitted by the copyright and related rights legislation that applies to your use. For other uses you must obtain permission from the rights-holder(s) directly, unless additional rights are indicated by a Creative Commons license in the record and/ or on the work itself. This Thesis has been accepted for inclusion in WVU Graduate Theses, Dissertations, and Problem Reports collection by an authorized administrator of The Research Repository @ WVU. For more information, please contact researchrepository@mail.wvu.edu. 


\title{
A PRELIMINARY STUDY EVALUATING POTENTIAL PROBIOTIC USE IN ENDODONTICS
}

By

\author{
Khaled Seifelnasr, BDS, DDS
}

Thesis submitted to the School of Dentistry at West Virginia University in partial fulfillment of the requirements for the degree of

\author{
Master of Science \\ In \\ Endodontics
}

John G. Thomas, PhD, Chair

Anthony T. Borgia, DDS, MHA

Richard Jurevic, DDS, MSD, PhD

Department of Endodontics

Morgantown, West Virginia

2014

\section{Keywords:}

Probiotics, Dental, Endodontics, Candida albicans, Enterococci faecalis 


\title{
$\underline{\text { Abstract }}$ \\ A PRELIMINARY STUDY EVALUATING POTENTIAL PROBIOTIC USE IN ENDODONTICS
}

\author{
Khaled Seifelnasr, BDS, DDS
}

\section{Introduction:}

The main goal of endodontics is the prevention of apical periodontitis. This condition is the result of persistent pathogenic microorganisms such as Enterococcus faecalis (E. f) and Candida albicans (C.a) remaining in the root canal systems of teeth, and the ability of those organisms to directly cause acute and chronic inflammation in the periapical tissues. The concept of the use of probiotics has not yet been evaluated in addressing endodontic disease, but probiotics have proven successful in treating periodontal disease. Taking these findings into account, this preliminary work was performed to evaluate the possible effectiveness of a probiotic cocktail in preventing the growth of two potential endodontic patogens, Enterococcus faecalis and Candida albicans.

\section{Materials and methods:}

Five groups (I, II, III, IV and V) of commercial probiotics were selected and evaluated based upon numbers and concentration of organisms. Pathogenic test organisms were $C$. albicans (WVU Isolate Ca1028) and E. faecalis (WVU Isolate Ef01).

Phase 1 of the study was conducted by a disc diffusion assay test to evaluate zones of inhibition (ZOI) in millimeters (mm) of the selected probiotics against the E. $f$ and C.a. Microorganisms from probiotic samples were extracted via manufacturer's recommendations and mixed by weight. Thirty (30) microliters were then placed on sterile discs. Pathogenic organisms were set to a $1 \mathrm{McF}$ arland standard challenge. A five probiotic disc template on blood agar plates were inoculated with a lawn of either $E . F$ or $C$. $a$ and incubated at $37^{\circ} \mathrm{C}$ for 48 hours and 1 week. Two, five sterile disc templates with a lawn of either pathogenic organism were run parallel as a control.

Phase 2 was conducted by mixing $9 \mathrm{ml}$ of $30 \%$ poloxamer 407 and MRS broth in a test tube with $500 \mathrm{ml}$ of either E. for C. $a$ set at a $1 \mathrm{McF}$ arland standard, together with $500 \mathrm{ml}$ of either Group I or Group IV probiotic mixtures, set at a 2 McFarland standard. Samples were incubated at $37^{\circ} \mathrm{C}$ for 48 hours, followed by serial dilutions of $10^{-2}, 10^{-4}$, and $10^{-6}$ for evaluation of $\mathrm{CFU} / \mathrm{ml}$ counts. Controls were E. for C. a $30 \%$ poloxamer with MRS broth and no probiotics. 


\section{$\underline{\text { Results: }}$}

Phase 1: Based on a One-Way ANOVA analysis, Groups I, IV and V showed the most statistically significant results $(\mathrm{P}<0.05)$ with a Mean of $7.4 \mathrm{~mm}, 10.05 \mathrm{~mm}, 11.2 \mathrm{~mm}$ for $C$. $a$ and a Mean of $6.7 \mathrm{~mm}, 11.1 \mathrm{~mm}$, and $12.5 \mathrm{~mm}$ for E. $f$ respectively at 1 week, compared with no ZOI for the control. L. acidophilus, L. casei, L. rhamnosus and B. longum were all common strains in the probiotic cocktails selected.

Phase 2: Initial results showed a decrease to a $2 \log$ difference and a $1 \log$ difference for groups I and IV respectively regarding CFU/ml counts for C. $a$ and for E. f. Probiotic Groups I and IV showed complete elimination of $E$. $f$ and only probiotic colonies were present on observation.

\section{Conclusion:}

Recognizing that probiotics may act differently based on their composition and concentration, this study suggests that organisms such as L. acidophillus, L. rhamnosus, L.casei and B. Longum are effective for preventing the growth of E. faecalis and C. albicans in vitro against both their planktonic and biofilm morphological stages. Further evaluations for possible use in treating endodontic infection is suggested and warranted. Additionally, Phase 2 results of the study suggest that poloxamer 407 could be utilized as an ideal probiotic delivery vehicle when mixed with appropriate probiotics and utilized as an endodontic intra-canal medicament for treating teeth that have presented with non-vital pulps. 


\section{$\underline{\text { Dedication }}$}

I would like to dedicate this to my mother Amal, father Seifalla, wife Sumaya and children Aminah and Ammar, who have always instilled in me the idea that nothing is impossible and one can achieve anything in life as long as he puts thought and effort to accomplish it. Their love and care has always been the reason why I strive for success.

I would also like to dedicate this to both Dr.Borgia and Dr.Thomas for their mentorship, guidance, wisdom and most important of all, dedication, love and support.

\section{$\underline{\text { Acknowlegments }}$}

I would like to thank Dr.Jurevic for his support.

I would like to thank Chris waters for his help as well as the endodontic staff for their help during residency.

I would like to thank Dr's Nicholson, Parsa and Xu for making this residency fun and delightful, I wish all of them a successful and happy future. 


\section{TABLE OF CONTENTS}

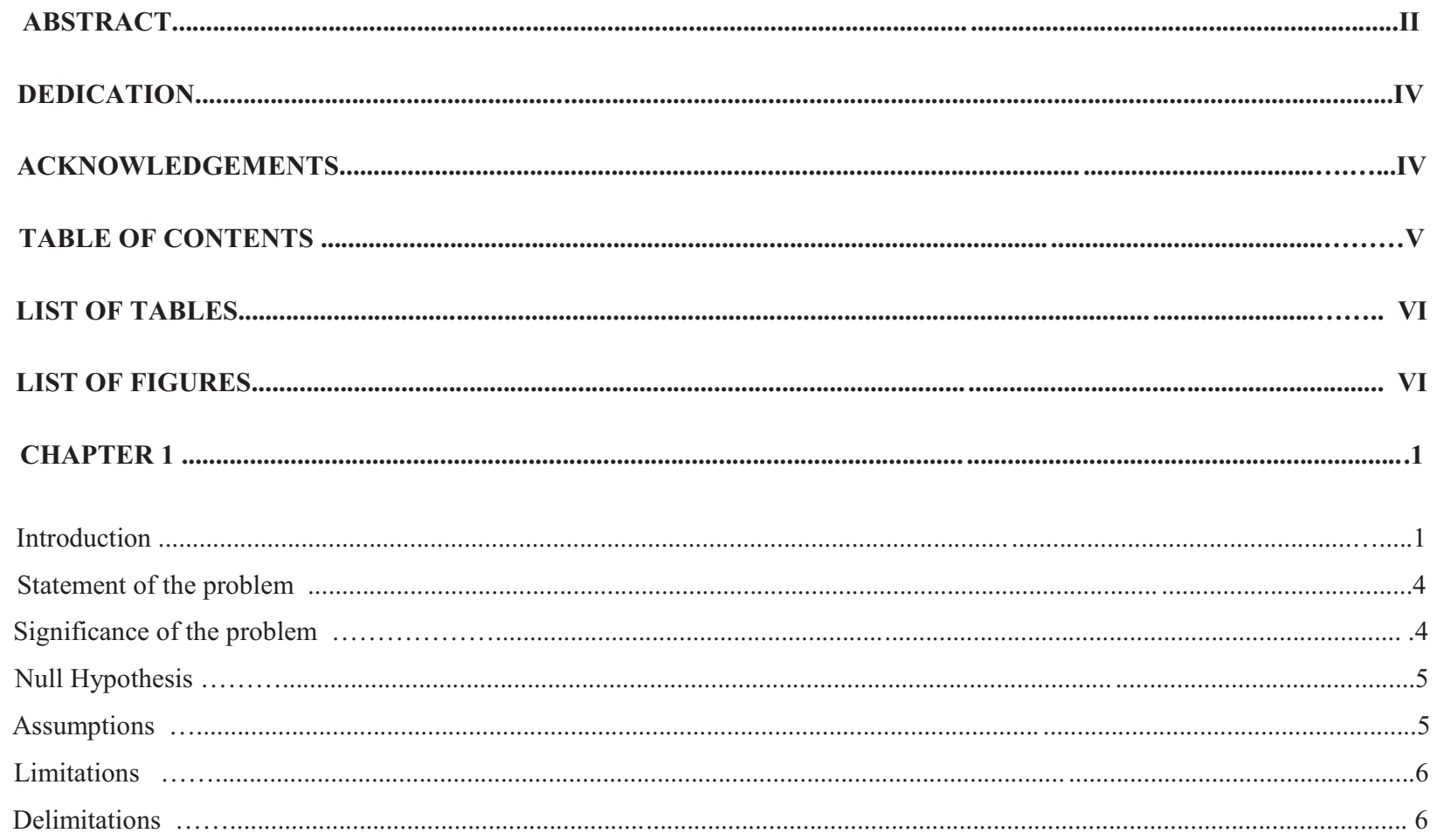

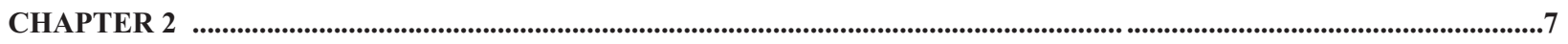

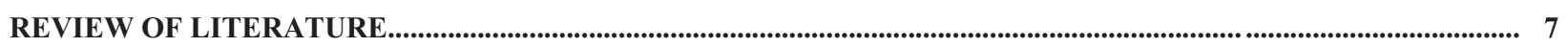

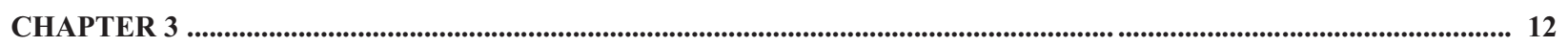

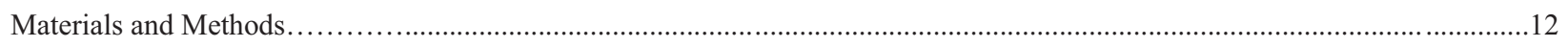

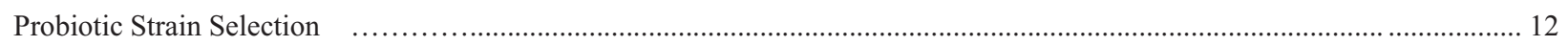

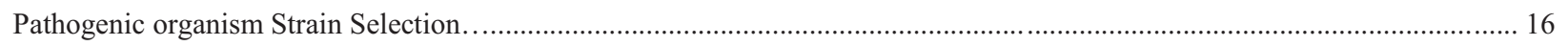

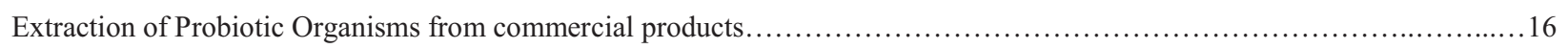

Phase 1: Testing for Probiotic efficacy against E.faecalis and C.albicans; Planktonic Stage evaluation............................ 24

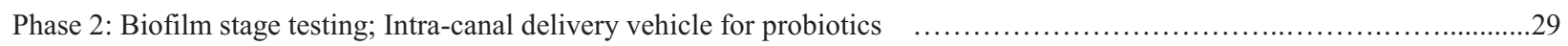

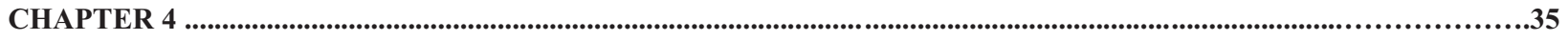

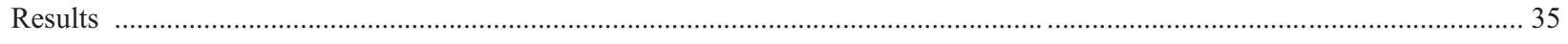

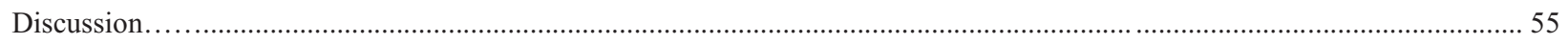

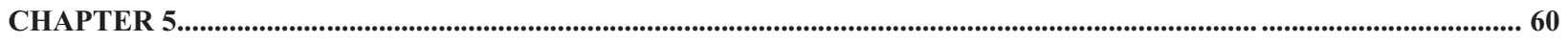

Conclusion $\quad$. .

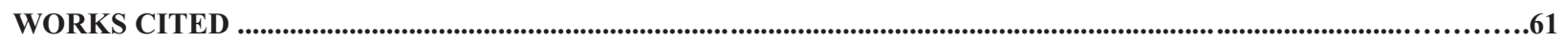




\section{$\underline{\text { List of Tables }}$}

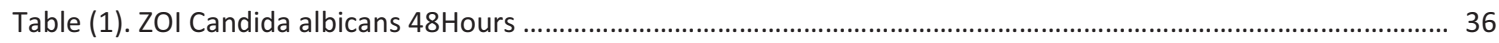

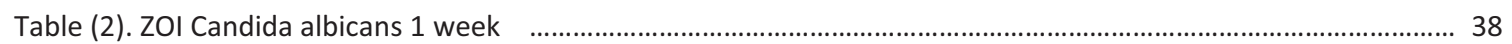

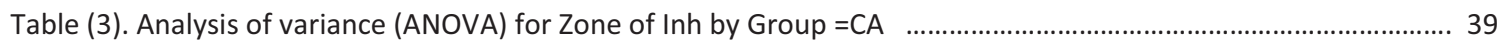

Table (4). Effect Tests for Zone of Inh by Group =CA

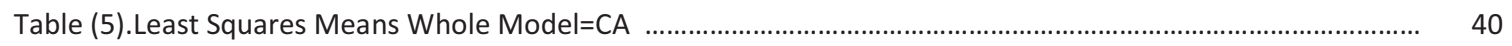

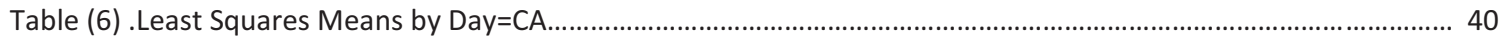

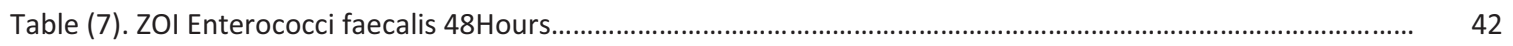

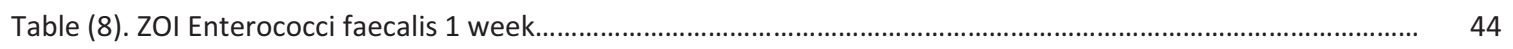

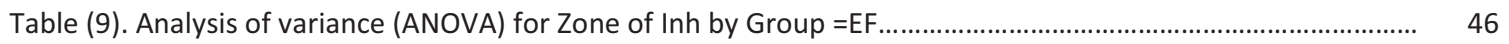

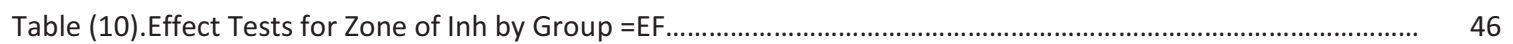

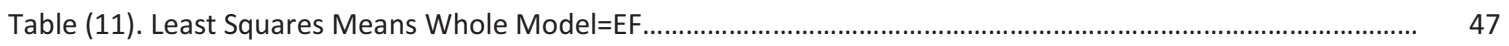

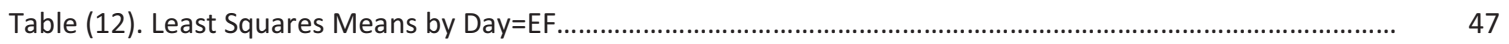

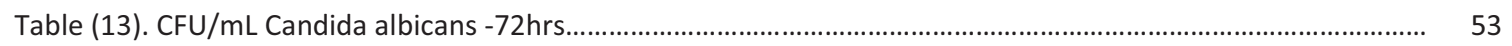

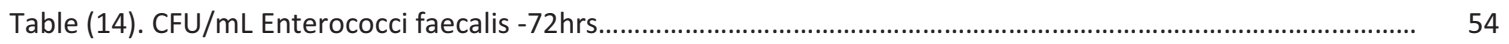

\section{List of Figures}

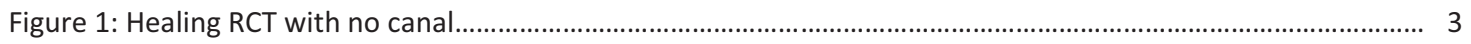

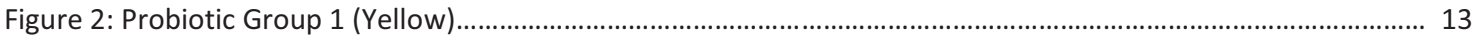

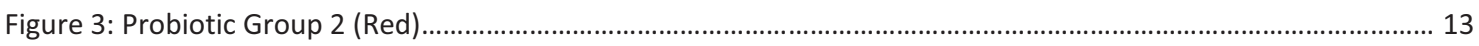

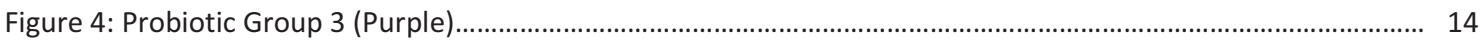

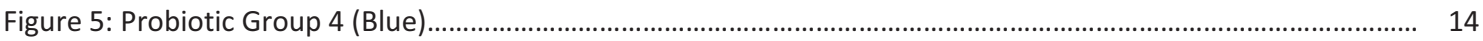

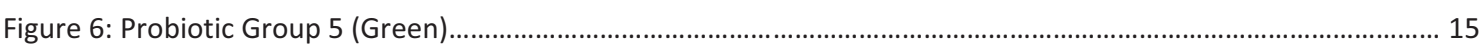

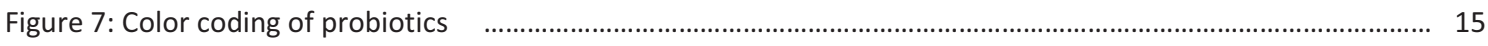

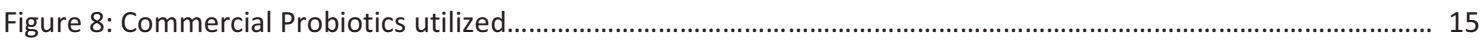


Figure 9: (A)L-spreader, (B) Micro-Pipette, (C) Sterile pliers and Loops, (D) Vortex machine...................................... 17

Figure 10: (A) Probiotics in TSB broth (posterior) and MRS broth(anterior), (B)Probiotics in TSB broth........................18

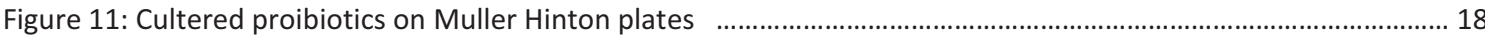

Figure 12: (A) C.albicans lawn on blood agar plate, (B) E.faecalis on blood agar plate............................................. 19

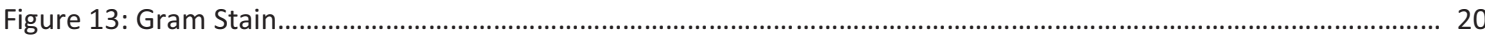

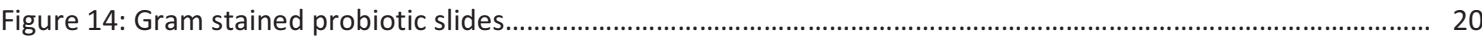

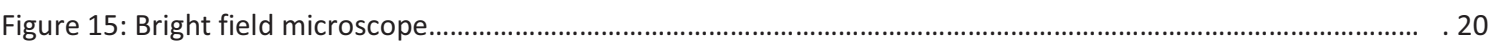

Figure 16: Live/Dead Stain slides prepared for fluorescent microscope ................................................................ 21

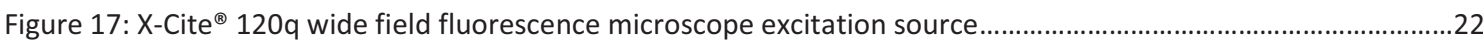

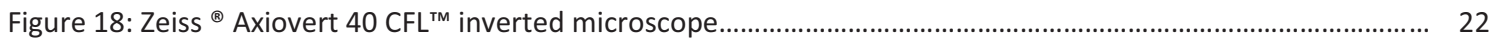

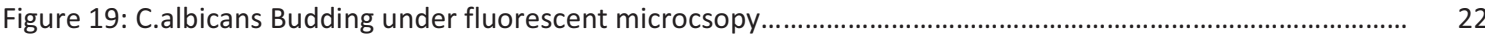

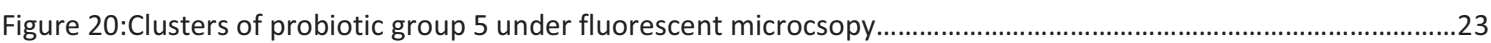

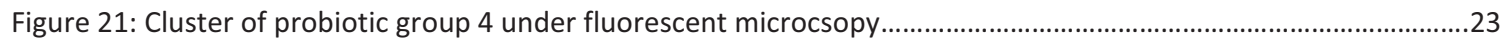

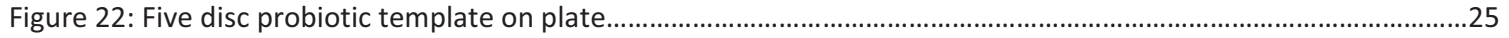

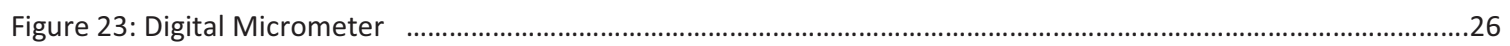

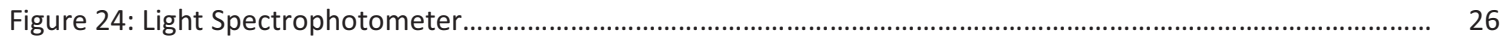

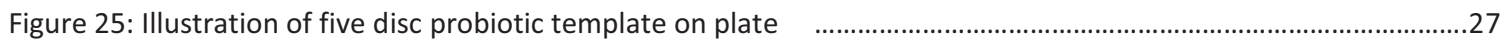

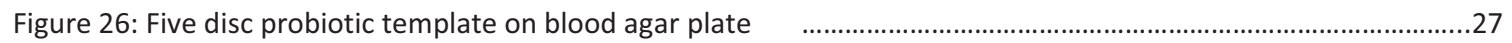

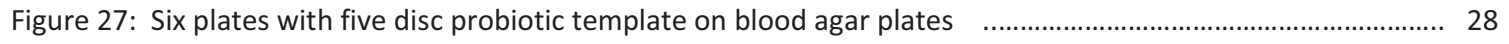

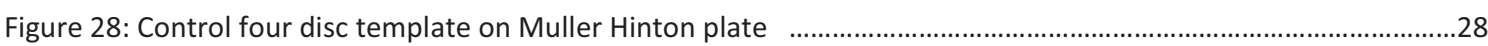

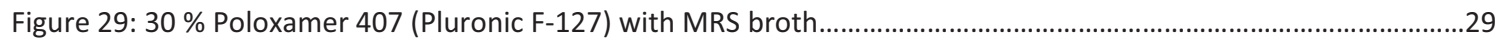

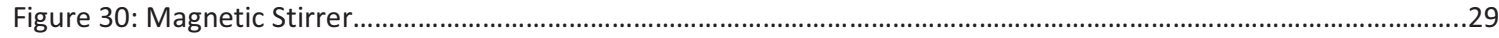

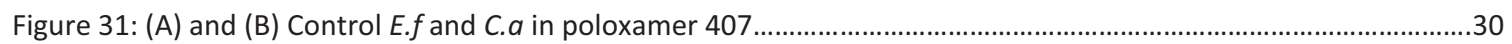

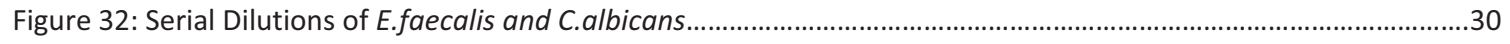

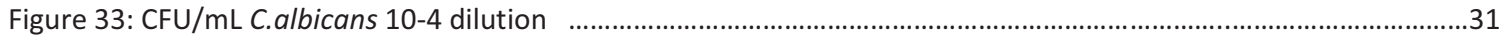

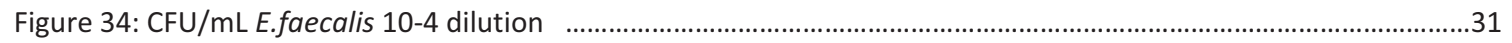

Figure 35: Poloxamer with pathogenic organisms and test probiotics 4 and 1 mixed together $\quad$..............................33

Figure 36: Serial dilutions of poloxamer/probiotic/pathogenic organisms mixed together .......................................33

Figure 37: Serial dilutions of poloxamer/probiotic/pathogenic organisms mixed together on blood agar plates..........34 
Figure 38: (A) and (B) ZOI's for Candida albicans 48-72hrs with five disc template on blood agar

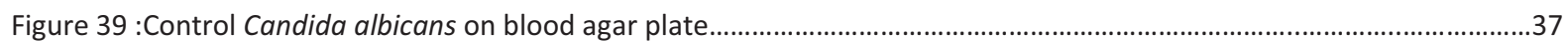

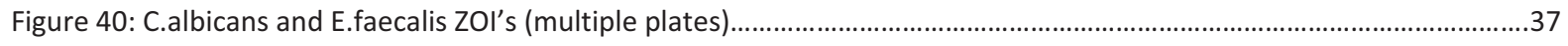

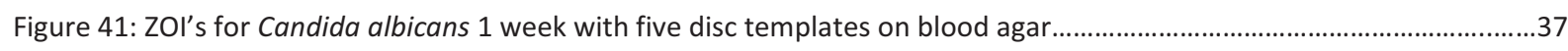

Figure 42: Zone of growth 24hrs for Candida albicans on blood agar

Figure 43: (A) and (B) ZOI's for Candida albicans one week with five disc template on blood agar .......................................39

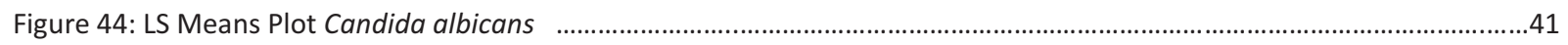

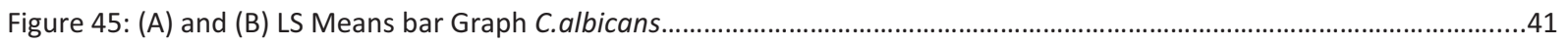

Figure 46: (A), (B) and (C) ZOI's for Enterococci faecalis 48-72hrs with five disc template on blood agar.............................42

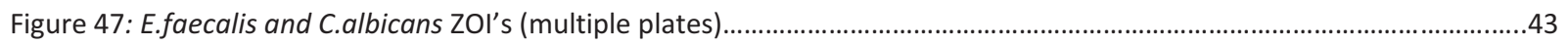

Figure 48: Zone of growth 24hrs for Enterococci faecalis on blood agar..............................................................................43

Figure 49: (A) and (B) ZOI's for Enterococci faecalis 48-72hrs with five disc template on blood agar...................................44

Figure 50: (A), (B), (C) and (D) ZOI's for Enterocci faecalis one week with five disc template on blood agar...........................45

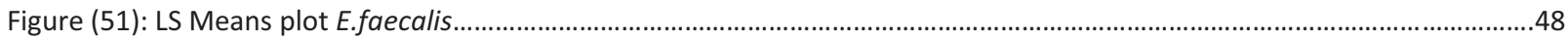

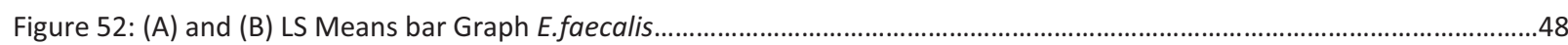

Figure 53: Poloxamer with pathogenic organisms and test probiotics 4 and 1 mixed together ...............................................50

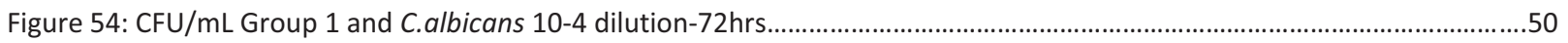

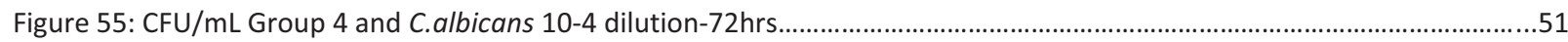

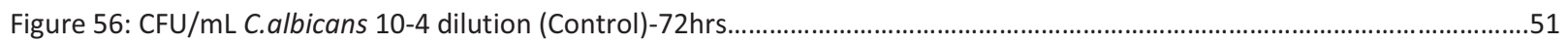

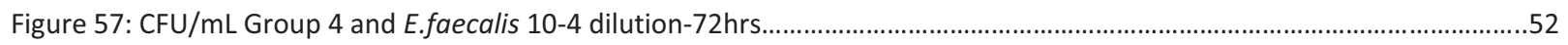

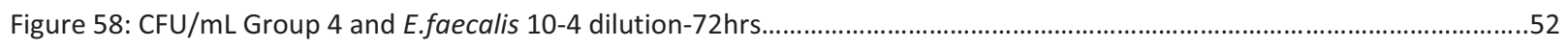

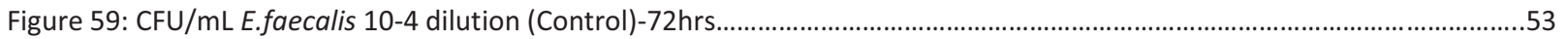

Figure 60: CFU/ml For Candida albicans (control), Group 1 and Group 4 mixed with Poloxamer-72hrs-Bar Graph..................54

Figure 61: CFU/ml For Enterococcus faecalis (control), Group 1 and Group 4 mixed with Poloxamer-Bar Graph .......................54

Figure 62: Poloxamer gel with group 1 in a syringe for intra-canal delivery ..............................................................................55 


\section{CHAPTER 1}

\section{Introduction}

Apical periodontitis is defined as inflammation and destruction of periradicular tissues caused by the presence of etiological agents of endodontic origin. ${ }^{(1)}$ It has long been known that these agents have been recognized as being either microorganisms or their metabolic products. $(1,2,3,6,7,8)$ It has been shown experimentally that no apical periodontitis develops in germ free rats, even when their mechanically exposed molar pulps are left in direct contact with the oral cavity, as opposed to control specimens with a conventional oral microflora, and in which massive periapical radiolucencies were observed. ${ }^{(2)}$

According to multiple studies, ${ }^{(1,3,4,5,6,7)}$ bacteria that normally inhabit the oral cavity have the ability to invade root canal systems during and after pulp necrosis. Microorganisms present in infected root canals are known to include a restricted group of species when compared to the rest of the normal flora found in the oral cavity. ${ }^{(1,3)}$ Most of the species that have been found in infected root canals have also been identified in periodontal pockets. ${ }^{(3)}$ Conditions exist in root canal systems that permit growth of anaerobic bacteria because they are capable of fermenting amino acids and peptides for metabolic needs. Bacteria that obtain energy mainly through the fermentation of carbohydrates have more restricted growth potential due to the lack of sufficient, appropriate and available nutrients in that specific environment. During the course of infection, interrelationships develop between microbial species, and microbial population shifts are produced as a result of these interactions. ${ }^{(1,3,4)}$ These microbial interactions play a significant role in the ecological regulation and eventual development of an endodontic habitat adapted polymicrobial flora. ${ }^{(1)}$

It has long been held that there are three basic principles that must be adhered to in clinical endodontics in order to achieve success in endodontic therapy. Also known as the "endodontic triad", these three principles are (1) thorough debridement of the root canal system, (2) sterilization of the root canal system, and finally (3) complete obturation of the root canal 
system. A key question in endodontics which continues to remain unanswered is, "Can pathogenic microorganisms actually be eliminated from an infected root canal'?

It has been proposed that it is impossible to obtain complete sterility within any given root canal system. Microscopic examination of serial sections of roots of many teeth have demonstrated the prevalence of multiple accessory and lateral canals. ${ }^{(9)}$ It is currently believed that these branches and ramifications can never be either completely debrided of tissue or properly rendered sterile. It is recognized and acknowledged that all that can be achieved is a reduction in the number of microorganisms in the main canal, or in other words, a reduction in the so called "bioload". Any perceived clinical success obtained from treatment of teeth with known positive root canal cultures can probably be ascribed to a reduction in the number of microorganisms, removal of most inflamed or necrotic tissue, and a favorable systemic background. ${ }^{(1,9)}$ Findings from multiple studies lead to speculation that there is a missing link or some unknown etiological factor in endodontic theory and practice.

This "missing link" could be an ongoing misunderstanding and even possibly incorrect concept of endodontic infection, with that thinking being restricted to the belief that all microorganisms must be removed from the root canal system, regardless of their pathogenicity or other characteristics. Rather, and in light of current and emerging findings in microbiology, it now seems reasonable that a better approach to addressing and dealing with microbial infection should be to maintain a state of equilibrium within the "Human Microbiome". The "Human Microbiome" is defined as "the recognized, normal microbial component of all humans and animals which is needed for health."(27,28) Multiple studies have demonstrated that the human microbiome is a necessary component for the health of the host, and that alterations in its ecological equilibrium can lead to disease; therefore, logic suggests that it is necessary to maintain a continuous state of equilibrium between these diverse microbial communities in order to maintain health. ${ }^{(27,28)}$

Accepting that the dentition is a part of the Oral Human Microbiome, it is proposed that there should be, of necessity, healthy organisms (probiotics) associated with the teeth in order to establish endodontic health, since complete sterility is impossible anywhere within the oral cavity. Therefore, to maintain or restore the equilibrium of the endodontic infrastructure, the 
host could be provided with microbiota which would then produce beneficial effects, shifting any deficiencies to a more favorable ecological system.

"Probiotics", as defined by the World Health Organization, are, live microorganisms which, when administered in adequate amounts, confer a health benefit on the host". Probiotics have been successfully used to control gastrointestinal diseases and appear to act through colonization resistance and/or immune modulation. ${ }^{(27,28)}$

Recently, probiotics have been introduced to dentistry for the treatment or prevention of disease. Experimental studies and clinical trials have demonstrated that certain gastrointestinal bacteria may control the growth of some oral microorganisms, including those cariogenic species associated with dental decay. Probiotics might potentially provide a means of preventing dental caries. $^{(10,11,14,17,19)}$ The oral administration of probiotics has also been explored in the control of periodontal disease by reducing plaque levels and gingival inflammation. ${ }^{(1,24)}$

The purpose of this study is to evaluate the potential use of probiotic therapy as an adjunct in endodontic therapy along with its effect on the reduction or elimination of apical periodontitis.
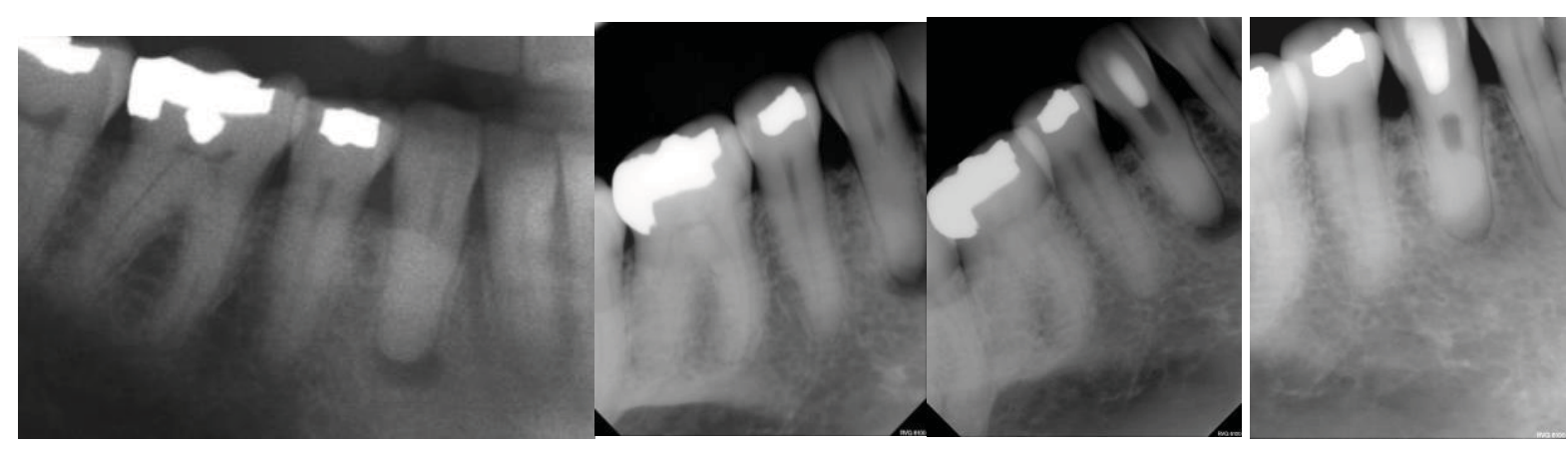

\section{Figure 1: Healing RCT with no canal}




\section{Statement of the Problem}

Does the use of probiotics have the potential to outcompete or eliminate pathogenic microorganisms in endodontic therapy?

\section{Significance of the Problem}

There is a need for an innovative or novel approach to the current treatment modalities which can possibly result in a higher, long term success in endodontic therapy. Despite the universal and widely spread advancement of technology throughout all facets of dentistry, the same basic approach, in conjunction with the same materials, has been employed over the past several decades in providing endodontic treatment. The concept of the "Human Microbiome" has been neglected in the development of new and better strategies used in endodontic therapy, even though it is now known to be an integral and undeniable part of human health. The novel concept in endodontics that the intentional establishment of a microbial equilibrium inside the root canal system by utilizing probiotics, a procedure which might result in improved overall success rates in the reduction of apical periodontitis, needs to be carefully examined and explored. As previously mentioned, the use of probiotics has gained universal acceptance by the gastroenterology community and some probiotics have been shown to be successful in treating certain gastrointestinal diseases. The concept of using probiotics has recently been utilized for the prevention of both dental caries and periodontal disease. Periodontal disease is known to have a number of pathogenic microorganisms in common with those found in endodontic infections, and therefore utilizing probiotics within the root canal system may also be found to be beneficial in endodontics. 


\section{Null-Hypothesis}

There is no significant difference between probiotic therapy and no therapy in eliminating or decreasing the amount of Enterococci faecalis and Candida albicans in planktonic and biofilm microbial stages when tested in an in vitro model.

\section{$\underline{\text { Assumptions }}$}

1. The Human Microbiome theory is gaining acceptance in medicine but has not yet been evaluated in endodontics.

2. Maintaining or restoring equilibrium with probiotics may show promising results in endodontic therapy.

3. Although sterility of the endodontic system is deemed necessary for endodontic success, achieving complete sterility is currently impossible under normal conditions.

4. Probiotics used against test organisms evaluated in vitro in both planktonic and biofilm stages in terms of measurement of zones of inhibition (ZOE) is an acceptable method of evaluation of the efficacy of the probiotics against pathogenic organisms.

5. Synergistically acting probiotics tested against pathogenic organisms may give a broader understanding of which particular species to select when conducting future studies.

6. Gram staining is an effective method to evaluate which organisms are present in blood agar/MRS or Muller Hinton plates.

7. Live/dead staining is an appropriate method of identifying probiotic activity.

8. Poloxamer 147 mixed with MRS broth and probiotics is a novel delivery vehicle for the introduction of probiotics into the root canal system as an intra-canal medicament. 


\section{$\underline{\text { Limitations }}$}

1. Due to financial limitations, commercial probiotics were utilized, and probiotic organisms were extracted either in groups or individually, according to manufacturer's instruction.

2. Due to time limitations, experiments were not performed in teeth.

3. As in any experimentation, human error may exist.

4. There is no known standardized method to identify different strains of microorganisms mixed together in a single group.

5. There have been no studies involving the use of probiotics in endodontics. The effective CFU count needed to eliminate or out compete the pathogenic organisms was unknown.

\section{$\underline{\text { Delimitations }}$}

1. Manufacturer was contacted to determine the method for extraction of probiotic species from commercial samples.

2. 50 Muller Hinton plates were utilized for initial evaluation.

3. 100 blood agar plates were utilized for evaluation of probiotic mixture against pathogenic organisms.

4. Probiotics and pathogenic organisms E. feacalis and C. albicans were tested in both planktonic and biofilm stages. 


\section{Chapter II}

\section{$\underline{\text { Review of Literature }}$}

The microbial component of humans and animals has been termed the indigenous microbiota. Experimental evidence shows that the microbiome is necessary for the health of the host, and that alterations in the ecological equilibrium of these microbes can lead to disease. ${ }^{(26)}$ It is therefore logical to expect that the introduction of microbes that are also members of the microbiome into an area of interest could help restore an ecological balance. ${ }^{(26)}$

A proposed solution to maintaining or restoring equilibrium would be to provide the host with microbiota which would produce perceived beneficial effects, shifting any deficiencies to a more favorable ecological system. The term "probiotics" is defined by the World Health Organization as live microorganisms which, when administered in adequate amounts, confer a health benefit on the host. The word "probiotics" was derived from the Greek, meaning "for life". The concept of probiotics is not new, but rather dates back to 1908, when Nobel Prize winner, Ukrainian bacteriologist Ilya Metchnikoff, suggested that the long life of Bulgarian peasants resulted from their consumption of fermented milk products which contained lactobacillus. ${ }^{(11,12,13,14,15,16,17,18,19,20,21,22,23,24)}$

Another solution to maintaining and/or restoring microbial equilibrium would be to administer substrates that improve the growth or metabolic activities of specific indigenous organisms, or so called, "prebiotics". The term prebiotic was introduced by Gibson and Roberfroid who exchanged "pro" for "pre", meaning "before". They defined prebiotics as a "non-digestible food ingredient that beneficially affects the host by selectively stimulating the growth and/or activity of one or a limited number of bacteria in the colon". ${ }^{(18)}$ Experimental models, along with several human studies, have shown that food ingredients, products, and supplements demonstrating a prebiotic effect, have been shown to modulate certain 
immunological biomarkers and affect activities of the immune system by inducing change in the gut microbiota. ${ }^{(29)}$

The term "synbiotic" is used when a product contains both probiotics and prebiotics. According to this approach, a food or food supplement will include not only live cells of the beneficial bacteria, but also their selective substrates, with the idea being that beneficial bacterial cells can grow quickly and competitively because of the presence of the selective substrate which allows it to predominate in the proposed environment. ${ }^{(18)}$

The introduction of these probiotics, prebiotics and synbiotics are coined as "Bacteriotherapy". Bacteriotherapy has been investigated in multiple studies to control infectious diseases, especially gastrointestinal disease, with the objective being the restoration and balance of the human microbiome. ${ }^{(14)}$

The suggested mechanisms of probiotic action on oral health are drawn from gastrointestinal studies. These several mechanisms include but are not limited to, immune modulation, down regulation of inflammatory responses, production of antimicrobial substances such as peroxides, organic acids and bacteriocins, mucin production, inhibition of epithelial invasion by inhibition of pathogens mucosal adherence, stimulation of IgA, and competition with other flora, including potential pathogens. ${ }^{(12,13,14,17,18,19)}$

Ideal features of a good probiotic would be:

(a) It should be a strain which is capable of exerting beneficial effect to the host

(b) It should be non-pathogenic and non-toxic

(c) It should be present as viable cells, preferably in large numbers

(d) It should be capable of surviving in the host environment

(e) It should also be able to maintain genetic stability in oral micro flora

(f) It should be stable and capable of remaining viable for periods under storage and field conditions. ${ }^{(12,13,14,17,18,19)}$

The most common probiotic bacteria belong to the Lactobacilli and Bifidobacteria genera, but certain strains of Streptococci have also been investigated. ${ }^{(22)}$ The reasoning behind 
why Lactobacillus species were chosen for this experiment is because they aide in producing those enzymes which digest and metabolize proteins and carbohydrates. They also aid in the synthesis of vitamins B and K, facilitating the breakdown of bile salts. Additionally, they have the ability to help enhance innate and acquired immunity, along with inhibiting proinflammatory mediators. Lactobacilli are considered to be a genus of gram positive facultative anaerobic microorganisms, with more than 100 species identified. Most notable are the strains L. acidophilus, L. salivarius, L. rhamnous, L. brevis, and L. casei which are utilized as probiotics. $^{(14)}$

Another organism as mentioned above is the Bifidobacterium species which are strictly gram positive anaerobes and which are the dominant organism found in the large intestine. Over 30 species of Bifidobacterium have been identified. Their characteristics include metabolism of lactose, generation of lactic ions from lactic acid, vitamin synthesis, fermentation of indigestible carbohydrates, and production of beneficial short chain fatty acids. $(10,12,14,17,18,19)$ Other species such as Streptococcus thermophillus are the organisms used as the chief cultures in yogurt production, owing to their distinguishing benefits of metabolism of lactose and improving lactose intolerance, while also possessing antimicrobial activity. Saccharomyces boulardii is a non-colonizing lactic acid producing yeast. Their most preeminent feature is that they secrete proteases and other substances that break down bacterial enterotoxins. They also help in the enhancement of immune function and have been shown to be beneficial in helping with $C$. difficile management. ${ }^{(30)}$

Recently, probiotics have been introduced in dentistry as an adjunct for the treatment or prevention of oral diseases. Currently, probiotic therapy has been investigated in experimental studies and clinical trials in an attempt to establish equilibrium in the oral component of the human microbiome. This philosophy would eliminate microorganisms associated with disease by allowing others associated with health to evolve and predominate.

Experimental studies and clinical trials are beginning to show advancement in multiple fields of dentistry such as: 


\section{- Caries Control:}

In saliva, caries associated microbes such as Streptococcus mutans have been shown to be reduced in number after the consumption of products containing the probiotics Lactobacillus and Bifidobacteria. ${ }^{(11,21)}$

\section{- Periodontal disease:}

Initial studies suggested that the use of probiotics could enhance oral health by decreasing periodontal inflammation. ${ }^{(31)}$ Subsequent studies evaluating patients who presented with various forms of periodontal disease such as gingivitis, pregnancy gingivitis and periodontitis, showed significant recovery after treatment with a culture of the L. acidophilus strain in most patients. ${ }^{(32)}$ Another study evaluated probiotic strains including L. reuteri, L. brevis and L. casei which revealed an improvement in gingival health, as measured by decreased gum bleeding. ${ }^{(33)}$ Further studies evaluated L. reuteri, L. brevis and L. salivarius probiotic strains against inflammatory markers where $L$. reuteri showed decreased levels of pro-inflammatory cytokines in gingival crevicular fluid $^{(33)}$ and the use of L. brevis and L. salivarius decreased matrix metalloproteinase activity along with other inflammatory markers in saliva. ${ }^{(34)}$

\section{- Oral Candidiasis:}

A preliminary study investigated the probiotic bacteria L. acidophilus and $L$. fermentum in oral cavities, which resulted in a rapid decline in C. albicans after the intake of the probiotics. Further consumption led to an almost undetectable number of fungi in the oral cavity. (35) Another study evaluated L. rhamnosus and Propionibacterium freudenreichii ssp. shermanii for the effect on oral candida infection in humans. After 16 weeks of therapy, the number of high oral yeast counts decreased, but no changes were observed in mucosal lesions. ${ }^{(35) .}$

\section{- Halitosis:}

A few clinical studies have found probiotic strains effective for the treatment of oral or gut associated halitosis. The studied strains included Lactobacillus, E. coli Nisle, S. salivarius and Weissella confusa isolates. ${ }^{(36)}$ In endodontics, it has long been held that there are three basic principles that must be adhered to in order to achieve success. This is known as the endodontic triad where, if followed, the end result of endodontic treatment should be both clinical and radiographic success. These three "principles" are (1) 
thorough debridement of the root canal system, (2) sterilization of the root canal system, and (3) complete obturation of the root canal system. As previously stated, the question which still remains today is whether or not microorganisms can be eliminated from an infected root canal system.

It has been proposed that attaining complete sterility in any part of the human oral cavity is impossible. Histological examination of serial sections of the roots of many teeth have revealed the prevalence of multiple accessory and lateral canals. ${ }^{(9)}$ It is inconceivable that these branches can be either debrided properly or made completely free of bacteria. All that can be reasonably expected and achieved in conventional root canal therapy is a reduction in the number of microorganisms within the main canal. Any success obtained from treatment of teeth with positive root canal cultures can be ascribed to a reduction in the number of microorganisms, removal of most inflamed or necrotic tissue, and a favorable systemic background. ${ }^{(1,9)}$ Multiple pathogenic organisms have been attributed to endodontic failure, but the two organisms in particular most commonly associated with treatment failure are E. faecalis and C. albicans. ${ }^{(8)}$

E. faecalis is resistant to most of the intra-canal medicaments, particularly calcium hydroxide dressings. This is due to its ability to regulate internal $\mathrm{pH}$ with an efficient proton pump, as well as its ability to survive prolonged starvation. Although endodontic infections are considered to be polymicrobial in nature, it has been shown that E. faecalis is the pathogen of significance in most failing endodontic treatment cases. ${ }^{(37,38)}$

Microbiological and correlative electron microscopic studies have shown the presence of yeasts in canals of teeth with apical periodontitis. ${ }^{(1)}$ Candida albicans is the most frequently isolated fungus from root filled teeth with apical periodontitis. ${ }^{(3)}$

The literature shows that there is a need for an innovative method of handling endodontic infections other than the currently used methods. A promising approach would be to manage endodontic treatment as part of the human microbiome and utilize probiotics in the same manner that they are used for other oral conditions to reestablish equilibrium of healthy flora.

Probiotics testing should be done against both planktonic and biofilm stages, the rationale being that although planktonic organisms represent free floating and homogeneous microbial 
cells, there has been a paradigm shift showing a link between surface attached, heterogeneous microbial cells (biofilms) and microbial pathogenesis, which then leads to human infections. ${ }^{(39)}$ Biofilms are defined as highly structured communities of microorganisms that are either surface associated or attached to one another and which are enclosed within a self-produced, protective extracellular matrix (ECM). Biofilm formation provides protection from the environment, resistance of physical and chemical stress, metabolic cooperation, and a community based regulation of gene expression. ${ }^{(39,40,41)}$ These features allow organisms (bacteria and fungi) in biofilms to assume a stronger pathogenic potential than those solely in a planktonic state. ${ }^{(40)}$ There is also evidence showing a major role of fungi in biofilm formation and disease. ${ }^{(41)}$

\section{Chapter III}

\section{$\underline{\text { Materials and Methods }}$}

\section{- Probiotic Strain Selection:}

Due to financial limitations, individual probiotic strains were not able to be purchased from ATCC (American Type Culture Collection). Alternatively, commercial probiotic cocktails were purchased for utilization in this study, decreasing financial burden. After extensive research was conducted about effective probiotic strains used to establish equilibrium of the gut flora, five probiotic cocktails were purchased from Klaire Labs ${ }^{\circledR}$. The probiotic cocktails were delivered in wrapped ice packaging in order to preserve viability of the organisms. Upon arrival, the probiotics were stored in a refrigerator at $30^{\circ} \mathrm{F}$. Each group of probiotic blend was designated a specific color to easily identify the group for the study. The five groups (G I, GII, GIII, GIV and GV) of commercial probiotics were selected and evaluated based upon numbers and concentration of organisms. 


\section{The five commercial groups of probiotics were as follows:}

I. Group 1: Designated Color $=$ YELLOW

\section{Vital-Immune Biotic ${ }^{\circledR}$ Caps}

\begin{tabular}{|c|c|c|}
\hline Amount Per Capsule & & \% Daily Value \\
\hline $\begin{array}{l}\text { Probiotic Blend ( } 5+\text { billion CFUs) in } \\
\text { a baseof inulin (derived from } \\
\text { chicory root) }\end{array}$ & $460 \mathrm{mg}$ & * \\
\hline Lactobacillus rhamnosus & $\begin{array}{c}2.0+\text { billion } \\
\text { CFUs }\end{array}$ & * \\
\hline Lactobacillus casei & $\begin{array}{c}1.5+\text { billion } \\
\text { CFUs }\end{array}$ & $*$ \\
\hline Lactobacillus acidophilus & $\begin{array}{c}1.0+\text { billion } \\
\text { CFUs }\end{array}$ & $*$ \\
\hline Bifidobacterium longum & $\begin{array}{l}0.5+\text { billion } \\
\text { CFUs }\end{array}$ & * \\
\hline
\end{tabular}

Figure 2: Probiotic Group 1 (Yellow)

II. Group 2: Designated Color $=\mathrm{RED}$

$\underline{\text { ABX Support }}{ }^{\mathrm{TM}}$

\begin{tabular}{|lcc|}
\hline Amount Per Capsule & \multicolumn{1}{c|}{$\begin{array}{c}\% \text { Daily } \\
\text { Value }\end{array}$} \\
\hline $\begin{array}{l}\text { Probiotic Blend (10+ } \\
\text { billion CFUs) in abase of } \\
\text { inulin (derived from } \\
\text { chicoryroot) }\end{array}$ & $430 \mathrm{mg}$ & $*$ \\
Saccharomyces boulardii & $\begin{array}{l}5.0+ \\
\text { billion } \\
\text { CFUs }\end{array}$ & $*$ \\
\hline Lactobacillus rhamnosus & $\begin{array}{l}2.5+ \\
\text { billion } \\
\text { CFUs }\end{array}$ \\
\hline Bifidobacterium bifidum & $\begin{array}{l}1.25+ \\
\text { billion } \\
\text { CFUs }\end{array}$ \\
\hline Bifidobacterium breve & $\begin{array}{l}1.25+ \\
\text { billion } \\
\text { CFUs }\end{array}$ \\
\hline
\end{tabular}

Figure 3: Probiotic Group 2 (Red) 
III. Group 3: Designated Color $=$ PURPLE

\section{Sacchromyces Boulardii}

\begin{tabular}{l}
\hline Amount Per Capsule \\
Probiotic Blend in a base of cellulose $\quad 320 \mathrm{mg}$
\end{tabular}

$\underline{\text { Ther-Biotic }{ }^{\circledR} \text { Complete Powder }}$

\begin{tabular}{|c|c|c|}
\hline Serving Size 1/4 Teaspoon & & \\
\hline Amount Per $1 / 4$ Teaspoon & & $\begin{array}{l}\text { \% Daily } \\
\text { Value }\end{array}$ \\
\hline $\begin{array}{l}\text { Probiotic Blend ( } 100+\text { billion CFUs) in } \\
\text { a base of inulin (derived from chicory } \\
\text { root) and InTactic } ₫ \text { proprietary } \\
\text { polysaccharide complex }\end{array}$ & $1,000 \mathrm{mg}$ & $*$ \\
\hline Lactobacillus rhamnosus & $\begin{array}{c}24.0+ \\
\text { billion CFUs }\end{array}$ & * \\
\hline Bifidobacterium bifidum & $\begin{array}{c}20.0+ \\
\text { billion CFUs }\end{array}$ & $*$ \\
\hline Lactobacillus acidophilus & $\begin{array}{c}12.0+ \\
\text { billion CFUs }\end{array}$ & * \\
\hline Lactobacillus casei & $\begin{array}{c}10+\text { billion } \\
\text { CFUs }\end{array}$ & * \\
\hline Lactobacillus plantarum & $\begin{array}{c}8.0+\text { billion } \\
\text { CFUs }\end{array}$ & $*$ \\
\hline Lactobacillus salivarius & $\begin{array}{c}8.0+\text { billion } \\
\text { CFUs }\end{array}$ & $*$ \\
\hline Bifidobacterium longum & $\begin{array}{c}4.0+\text { billion } \\
\text { CFUs }\end{array}$ & * \\
\hline Streptococcus thermophilus & $\begin{array}{c}4.0+\text { billion } \\
\text { CFUs }\end{array}$ & $*$ \\
\hline Lactobacillus bulgaricus & $\begin{array}{c}4.0+\text { billion } \\
\text { CFUs }\end{array}$ & * \\
\hline Lactobacillus paracasei & $\begin{array}{c}2.0+\text { billion } \\
\text { CFUs }\end{array}$ & $*$ \\
\hline Bifidobacterium lactis & $\begin{array}{c}2.0+\text { billion } \\
\text { CFUs }\end{array}$ & * \\
\hline Bifidobacterium breve & $\begin{array}{l}2.0+\text { billion } \\
\text { CFUs }\end{array}$ & * \\
\hline
\end{tabular}

Figure 5: Probiotic Group 4 (Blue) 
V. Group 5: Designated Color $=$ GREEN

$\underline{\text { Vital-10® Powder }}$

\begin{tabular}{|lcc|}
\hline Amount Per 1/4 Teaspoon & $\%$ Daily Value \\
\hline $\begin{array}{l}\text { Probiotic Blend (10+ billion CFUs) in } \\
\text { a base of inulin (derived from chicory } \\
\text { root) }\end{array}$ & $1,000 \mathrm{mg}$ & $*$ \\
\hline Lactobacillus acidophilus & $*$ \\
\hline Bifidobacterium bifidum & $\begin{array}{c}3.7+\text { billion } \\
\text { CFUs }\end{array}$ & $*$ \\
\hline Proprietary Blend of: & $\begin{array}{c}1.4+\text { billion } \\
\text { CFUs }\end{array}$ \\
\hline Lactobacillus bulgaricus & $\begin{array}{c}4.9+\text { billion } \\
\text { CFUs }\end{array}$ \\
\hline Lactobacillus rhamnosus & \\
\hline Lactobacillus brevis & \\
\hline Streptococcus thermophilus & \\
\hline Lactobacillus casei & \\
\hline Lactobacillus salivarius & \\
\hline Lactobacillus plantarum & \\
\hline Bifidobacterium lactis & \\
\hline
\end{tabular}

Figure 6: Probiotic Group 5 (Green)

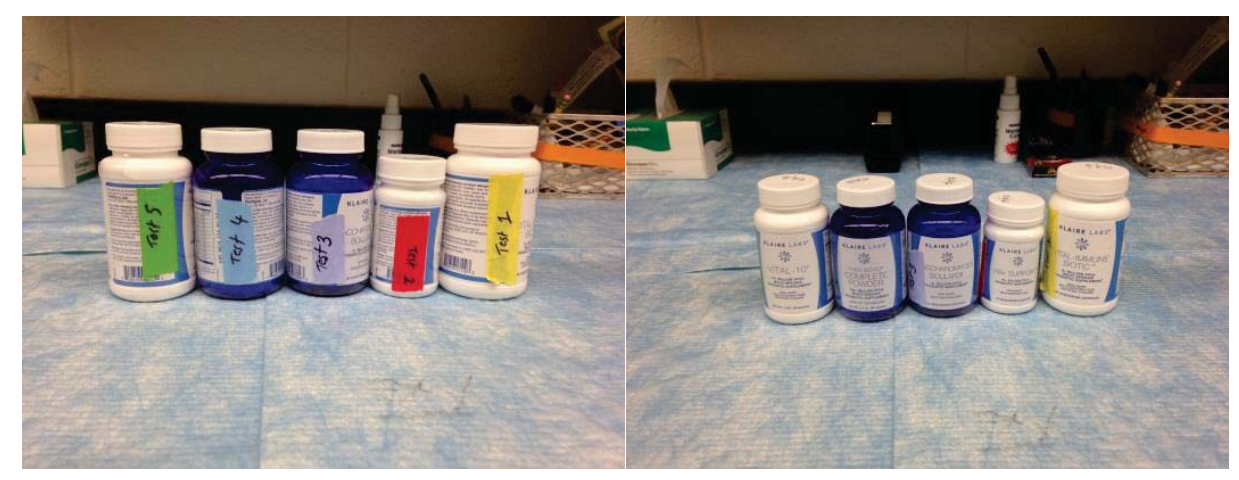

Figure 7: Color coding of probiotics Figure 8: Commercial Probiotics utilized 


\section{- Pathogenic Strain Selection:}

E. faecalis was chosen for this study after extensive literature review which had revealed that this organism possesses multiple properties leading to its key role as an endodontic pathogen. Some of these features: ${ }^{(37,38):}$

1. It is resistant to most of the intra-canal medicaments, particularly calcium hydroxide dressing due to its ability to regulate internal $\mathrm{pH}$ with an efficient proton pump.

2. E. faecalis can survive prolonged starvation.

3. Controlled studies have shown that E. faecalis is the pathogen of significance in most cases of failing endodontic treatment.

C. albicans was chosen as another pathogenic test organism due to: ${ }^{(1,3)}$

1. It's biphasic nature which allows it to be the universal co-aggregate in biofilms.

2. It is the most frequently isolated fungus from root filled teeth with apical periodontitis.

\section{- Extraction of Probiotic Organisms From Commercial Products:}

Klaire ${ }^{\circledR}$ Labs, a division of Prothera Inc ${ }^{\circledR}$, was contacted after purchasing and receipt of the probiotics that were intended for experimentation. The lab forwarded an extraction method for re-growing and culturing the microorganisms.

The following protocol was followed for the extraction/culturing method:

1. An aseptic protocol was followed for every extraction method via spraying the operative fields with $99 \%$ ethyl alcohol, followed by Cavicide ${ }^{\mathrm{TM}}$ spray or wipes. The operative fields were then left to dry. For manipulation of probiotics and extraction processes, sterile gloves were utilized to decrease any cross-contamination. All instruments such as sterile plastic pliers/loops were discarded after single use.

2.1.1 Grams of the dried probiotic powder of groups 1 through 5 were weighed aseptically on a lab scale. The measured powder was aseptically placed into sterile $15 \mathrm{ml}$ test tubes containing $10 \mathrm{ml}$ of sterile MRS broth. 
3. The tubes were then vortexed at room temperature on a vortex mixer (Fisher Scientific ${ }^{\mathrm{TM}}$ Digital mixer) for two minutes until the mixture was homogenous.

4. The samples were then kept at room temperature for 30 minutes to assure rehydration of the freeze-dried powder.

5. The samples were then returned to the vortex machine and vortexed for an additional two minutes.

6. Samples where incubated at $37^{\circ} \mathrm{C}$ in an incubator for 48 hours (an anaerobic chamber would have been preferred for growth of the microorganisms, but was unavailable).

7. Samples were then placed in the lab refrigerator at $4^{\circ} \mathrm{C}$ for no longer than two weeks before being used for testing to avoid any mutation in the test species. New probiotic stock was made every two weeks.

8. Samples that were to be utilized for testing were transferred via a sterile pipette from the stock solution into $9 \mathrm{ml}$ of MRS broth in sterile tubes and were adjusted to a McFarland Standard of 1 for standardization ( $1 \mathrm{McF}$ arland $\left.=3 \times 10^{8} \mathrm{CFU} / \mathrm{ml}\right)$. 9. To insure growth of microorganisms, $0.5 \mathrm{ml}$ of the $1 \mathrm{McFarland}$ mixture of the probiotic groups was transferred via a micropipette and spread on a blood agar plate or Mueller Hinton plates with an L-spreader, followed by incubation for 24 hours, 48 hours and 1 week. This was followed by gram staining and growth observation (CFU observation).

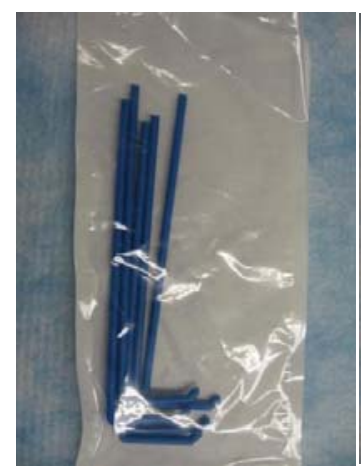

(A)

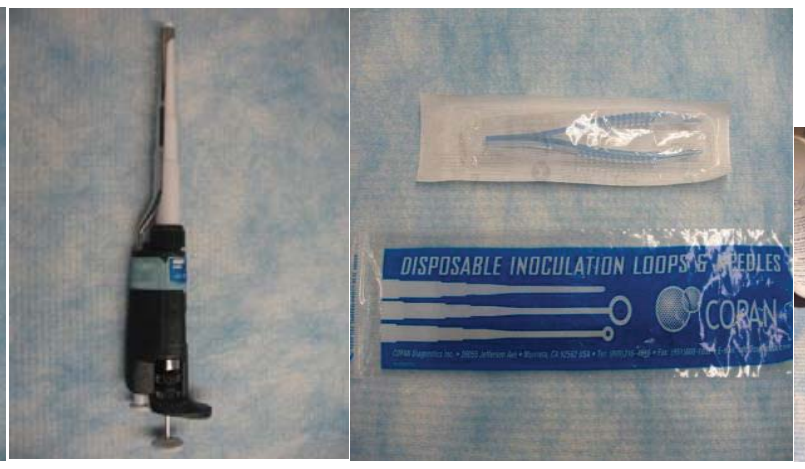

(B)
(C)

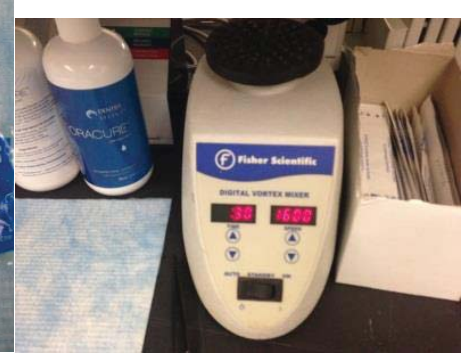

(D)

Figure 9: (A)L-spreader, (B) Micro-Pipette, (C) Sterile pliers and Loops, (D) Vortex machine 


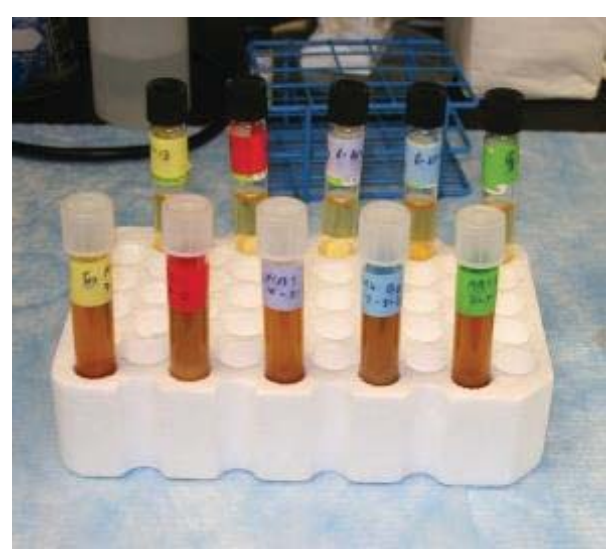

(A)

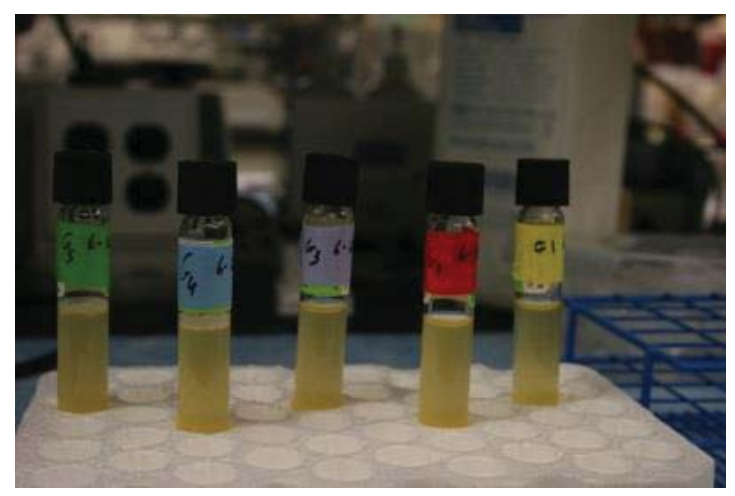

(B)

Figure 10: (A) Probiotics in TSB broth (posterior) and MRS broth(anterior), (B)Probiotics in TSB broth

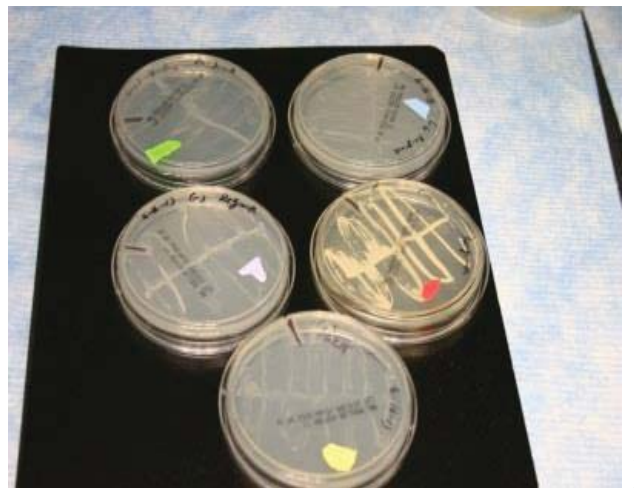

Figure 11: Cultered proibiotics on Muller Hinton plates 


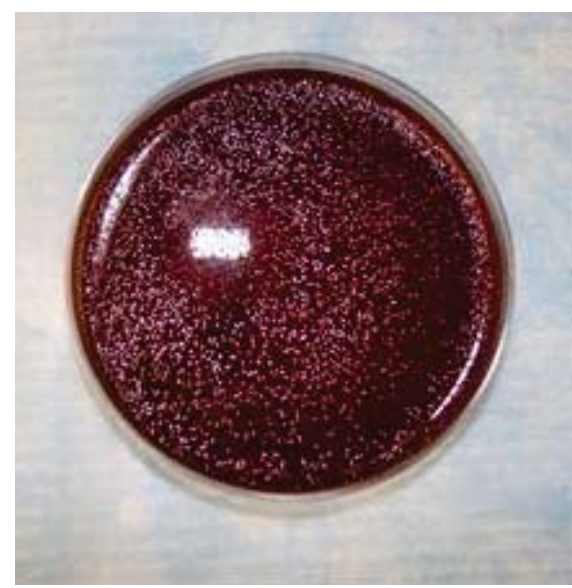

(A)

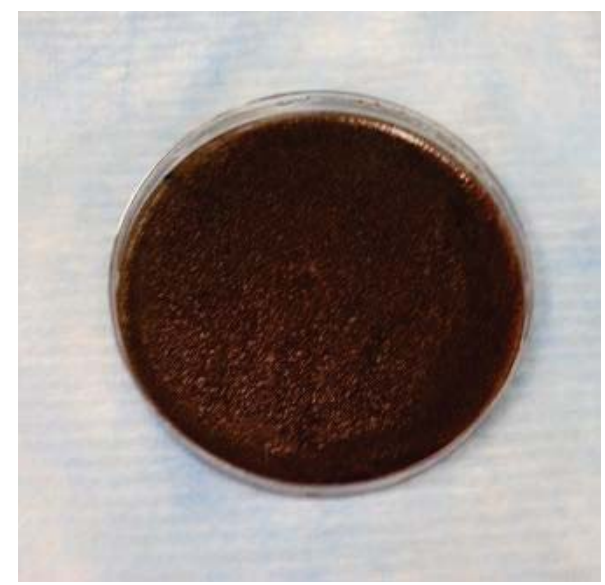

(B)

Figure 12: (A) C.albicans lawn on blood agar plate, (B) E.faecalis on blood agar plate

Steps done prior to the utilization of the probiotic blends to determine eligibility and presence of microorganisms prior to testing:

A. Gram staining was performed on each sample after plating and growth on blood agar plates. Gram staining process was as follows:

1. A $1 \mu \mathrm{L}$ sample was taken using a sterile plastic loop from the probiotic inoculated blood agar plate, and then smeared on to a wet microscope glass slide. All groups were tested (G1-G5).

2. The glass slide was heat fixed to dry the smear of cells.

3. Crystal violet staining reagent was applied for 1 minute.

4. The slide was washed in a gentle and indirect stream of tap water for 2 seconds.

5. Gram's iodine was applied for 1 minute.

6. The slide was washed in a gentle and indirect stream of tap water for 2 seconds.

7. Decolorizing agent was applied to the slide for 15 seconds until the slide was clear, followed by a gentle wash of the slide with tap water for 2 seconds.

8. Safranin was the applied for 30 seconds, followed by a final rinse of tap water for 2 seconds.

9. The slide was the left to air dry.

10. The results of the staining procedure were viewed using a Brightfield microscope. 
11. At the completion of the gram stain, it is noted that gram negative bacteria stain pink/red whereas gram positive bacteria stain blue/purple.

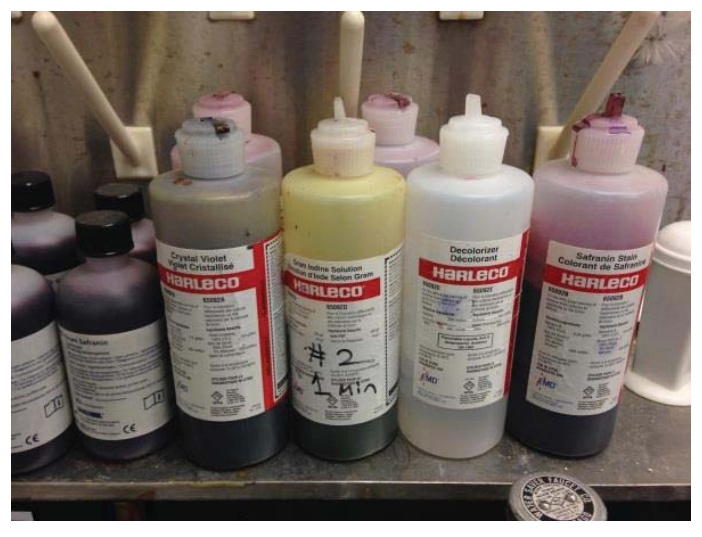

Figure 13: Gram Stain

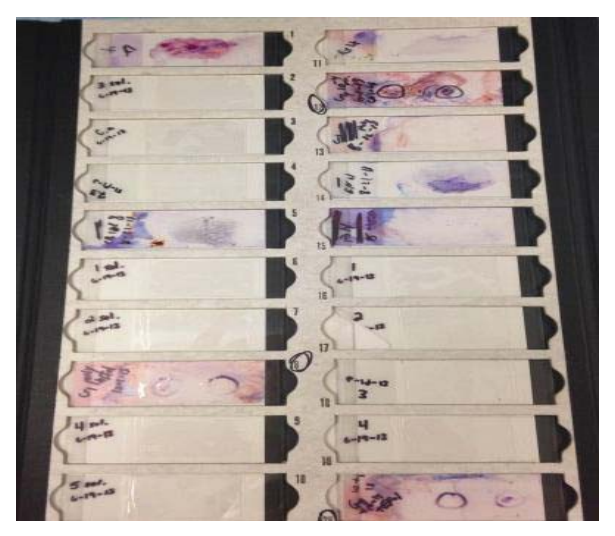

Figure 14: Gram stained probiotic slides

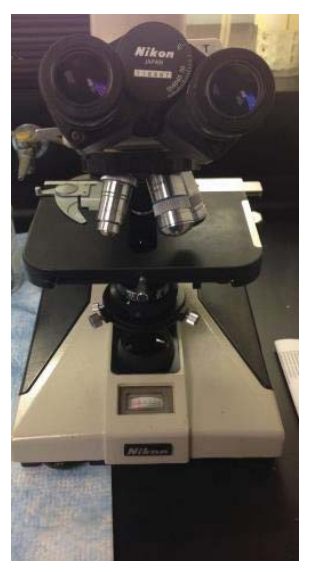

Figure 15: Bright field microscope 
B. Live/dead staining was performed by live/dead Baclight ${ }^{\mathrm{TM}}$ bacterial viability kits for evaluation of cell viability for all test organisms.

The following protocol was followed: Staining Bacteria in suspension with kit L13152

1. A $2 X$ stock solution of the live/deadD BacLight staining reagent mixture was prepared by dissolving the contents of one component A pipet (containing yellow-orange solids) and one component $\mathrm{B}$ pipet (containing red solids) in a common $5 \mathrm{~mL}-$ volume of filtersterilized $\mathrm{H}_{2} \mathrm{O}$.

2. A sample of the $2 \mathrm{X}$ stock solution was combined with an equal volume of the bacterial suspension. The final concentration of each dye will be $6 \mu \mathrm{M}$ SYTO 9 stain and $30 \mu \mathrm{M}$ propidium iodide.

3. The resulting solution was mixed thoroughly and incubated at room temperature in the dark for 15 minutes.

4. $5 \mu \mathrm{L}$ of the stained bacterial suspension was trapped between a slide and an $18 \mathrm{~mm}$ square coverslip.

5. Fluorescence was observed under a Zeiss ${ }^{\circledR}$ Axiovert $40 \mathrm{CFL}^{\mathrm{TM}}$ inverted microscope with $\mathrm{X}-\mathrm{Cite}{ }^{\circledR} 120 \mathrm{q}$ wide field fluorescence microscope excitation source.

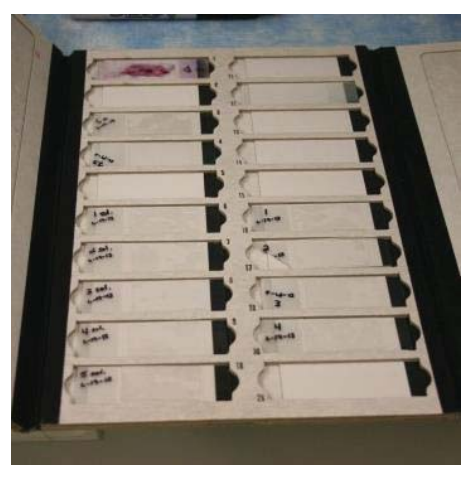

Figure 16: Live/dead stain slides prepared for fluorescent microscope 


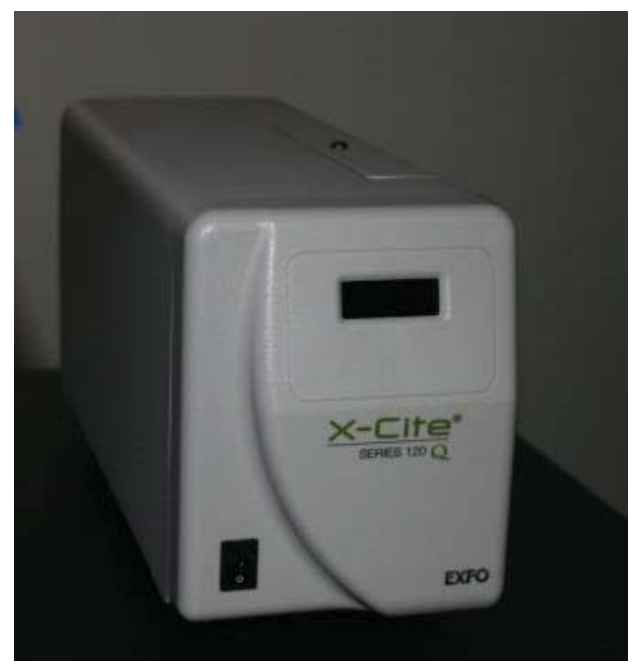

Figure 17: X-Cite ${ }^{\circledR}$ 120q wide field fluorescence microscope excitation source

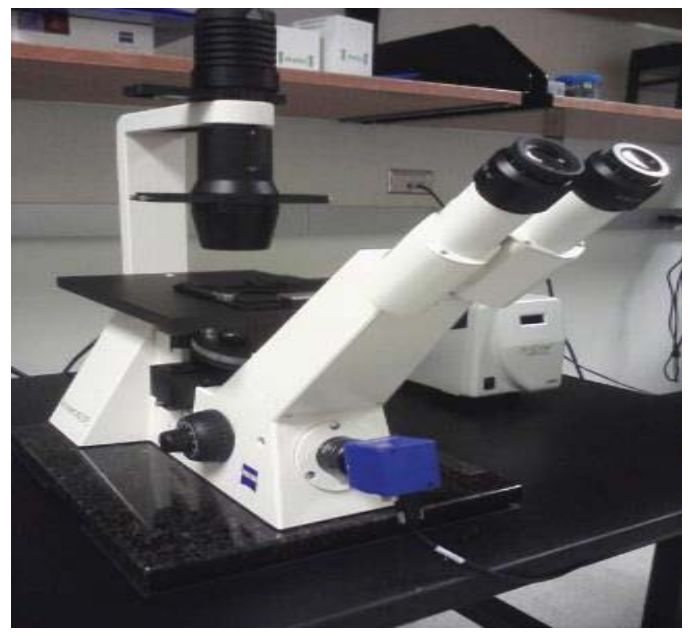

Figure 18: Zeiss ${ }^{\circledR}$ Axiovert 40 CFL $^{\mathrm{TM}}$ inverted microscope

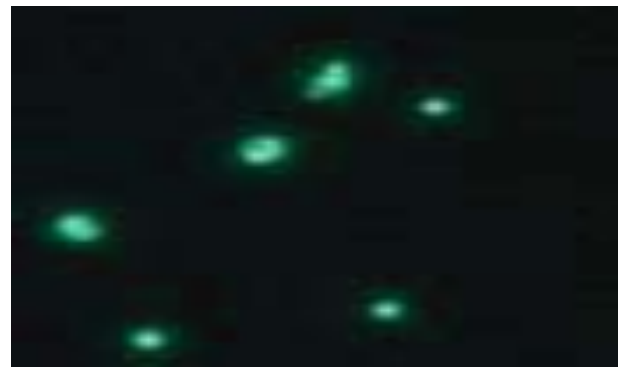

Figure 19: $C$. albicans budding under fluorescent microcsopy 


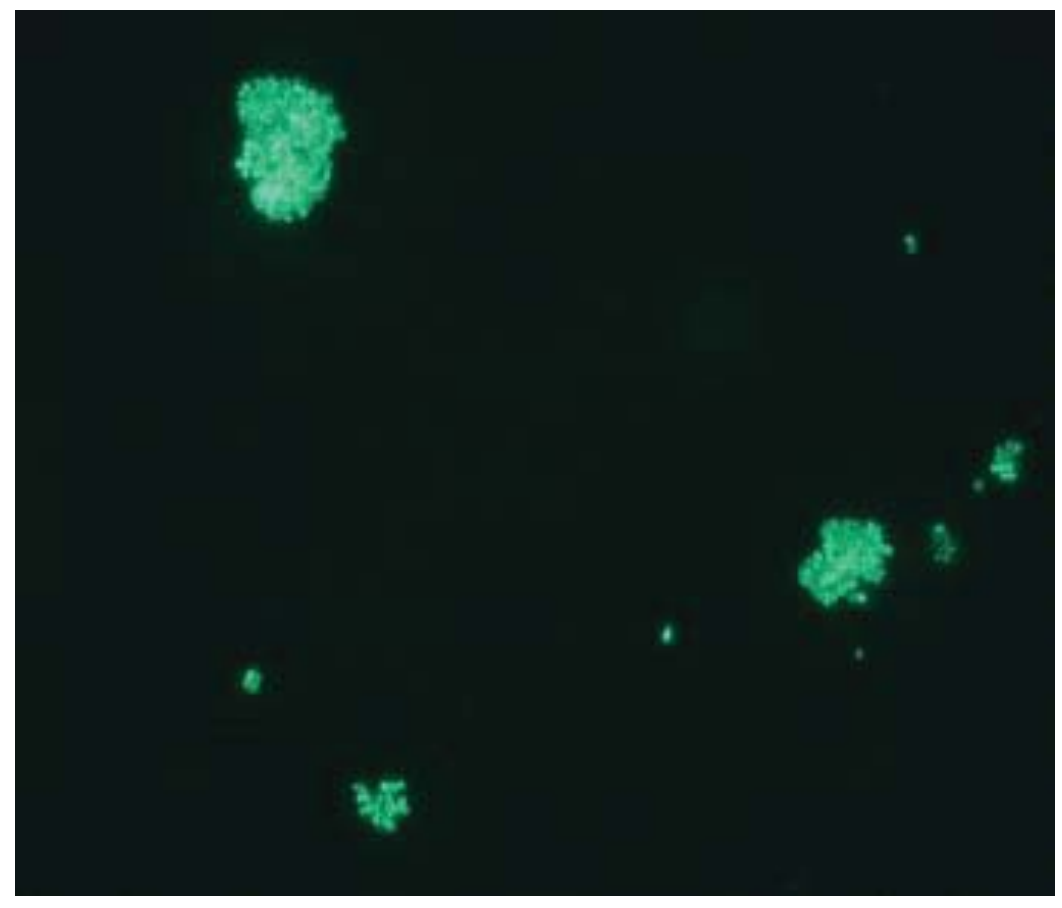

Figure 20:Clusters of probiotic group 5 under fluorescent microcsopy

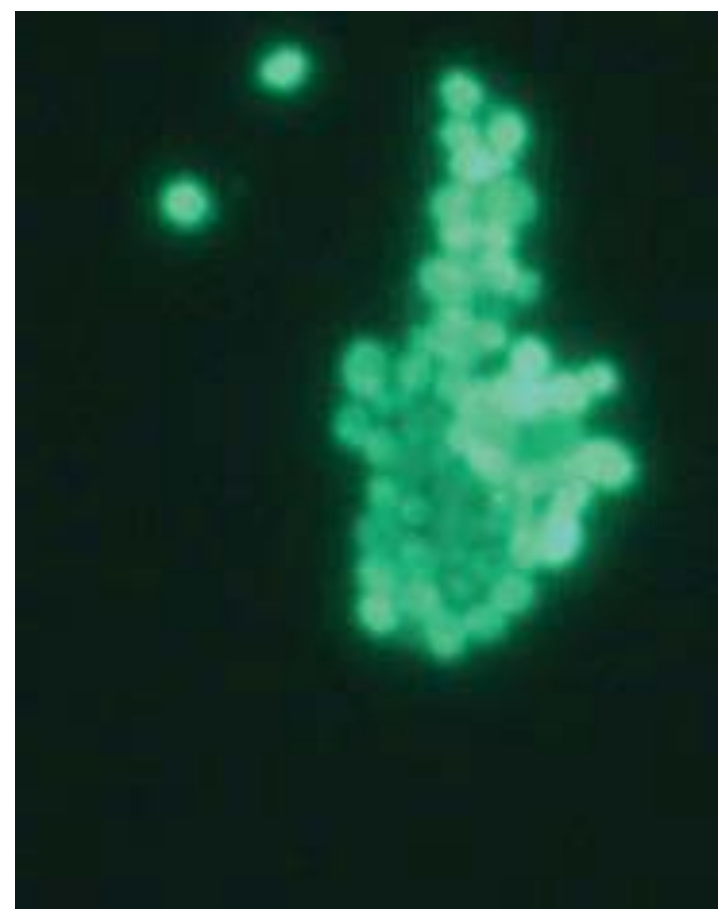

Figure 21: Cluster of probiotic group 4 under fluorescent microcsopy 


\section{Phase 1: Testing for Probiotic efficacy against $E$. faecalis and $C$. albicans; Planktonic Stage Evaluation.}

A disc diffusion assay test was conducted. The purpose of the Kirby-Bauer disc diffusion susceptibility test is to determine the sensitivity or resistance of pathogenic aerobic and facultative anaerobic bacteria to various antimicrobial compounds. In this study, probiotics where used to determine their antimicrobial effect against pathogenic organisms. The pathogenic organisms were grown on blood agar in the presence of test probiotic group impregnated filter paper discs. Observation of the presence or absence of growth around the discs is an indirect measure of the ability of that probiotic group to inhibit growth or out compete the pathogenic organisms E. faecalis or C. albicans.

Testing: An aseptic technique (sterile pipette carriers, alcohol and Cavicide wipes for surfaces, sterile cotton pliers, sterile loops and sterile L-spreaders) was utilized throughout the procedure to insure sterile environment, as well as prevention of cross contamination.

1. The five probiotic groups were extracted according to manufacturer's instructions and incubated for 48 hours.

2. Probiotic samples were then placed in $9 \mathrm{ml}$ MRS broth (De Man, Rogosa and Sharpe broth) and vortexed to insure a homogenous mixture, then set to a 2 McFarland standard via laser spectrophotometry (average $6 \times 10^{8} \mathrm{CFU} / \mathrm{ml}$ ).

3. The pathogenic organisms E. faecalis and C. albicans were freshly stocked, placed in 9 $\mathrm{ml}$ TSB (tryptic soy broth) and vortexed to insure homogenous mixture, then set to a 1 McFarland standard via laser spectrophotometry (average $3 \times 10^{8} \mathrm{CFU} / \mathrm{ml}$ ).

4. 500 microliters of E. faecalis was plated on $100 \mathrm{~mm}$ diameter blood agar plates and spread with a sterile L-Loop. The sample was incubated for 24 hours to allow growth of a bacterial lawn.

5. 500 microliters of C. albicans was plated on $100 \mathrm{~mm}$ diameter blood agar plates and spread with a sterile L-Loop. The sample was incubated for 24 hours to allow growth of a bacterial lawn. 
6. 20 microliters of probiotic G I through G V were placed on sterile blank paper discs and left for 15 seconds to allow the discs to saturate with the probiotic cocktails. The discs were then transferred to the previously grown lawns of the pathogenic test organisms, E. faecalis and C. albicans, according to a 5 group template as seen in figure (22).

7. The blood agar plates were then incubated at $37^{\circ} \mathrm{C}$ and evaluated at 48 hours and 168 hours (1 week), respectively.

8. The test was conducted three times per group against the organisms, E. faecalis and $C$. albicans to allow proper statistical analysis.

9. The control was conducted by growing E. faecalis and C. albicans lawns at a 1 McFarland standard with empty sterile discs placed on the lawns.

10. Results for ZOI's were measured with a digital micrometer in mm increments at 48 hours and 168 hours (1 week).

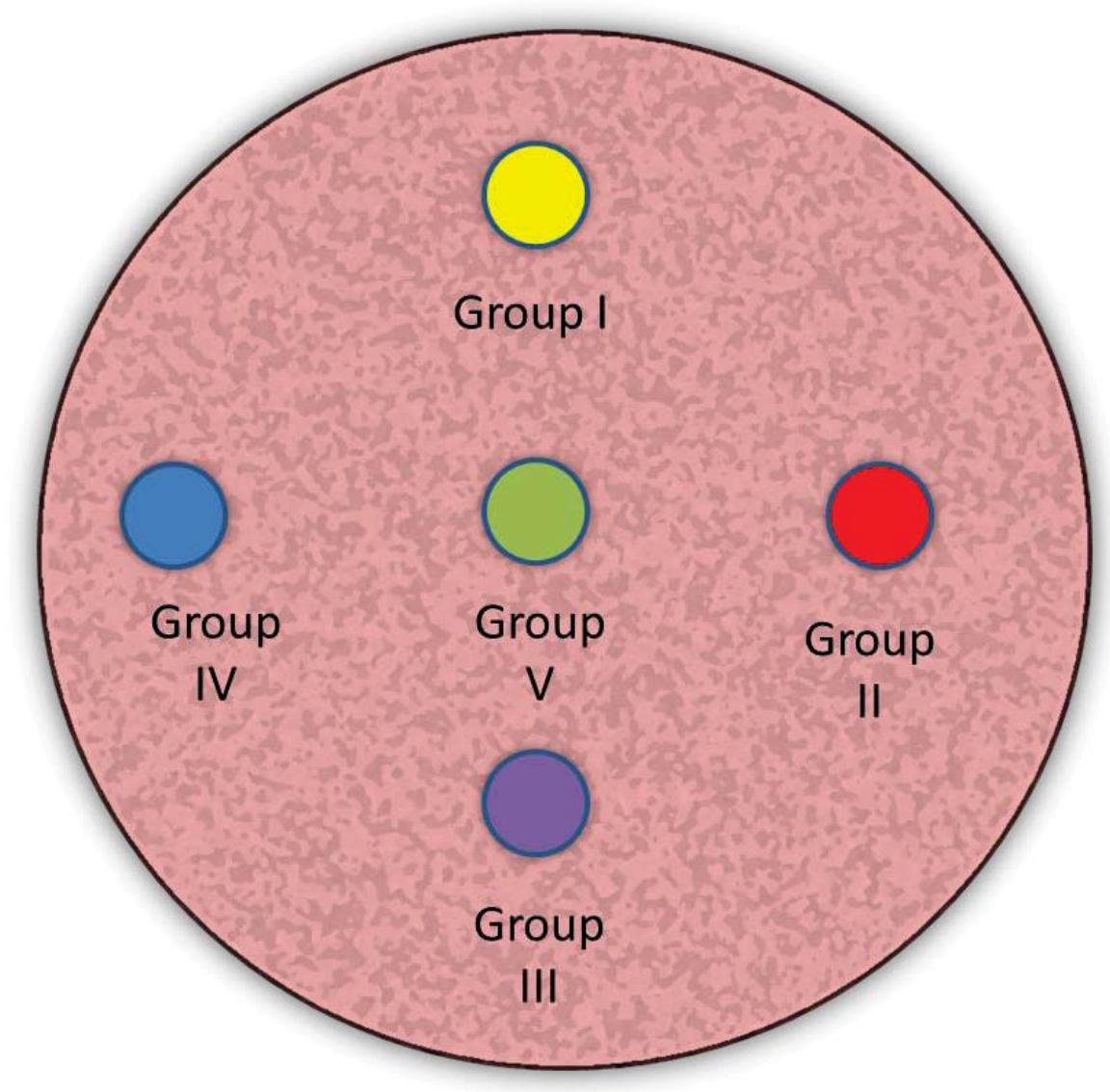

Figure 22: Five disc probiotic template on plate 


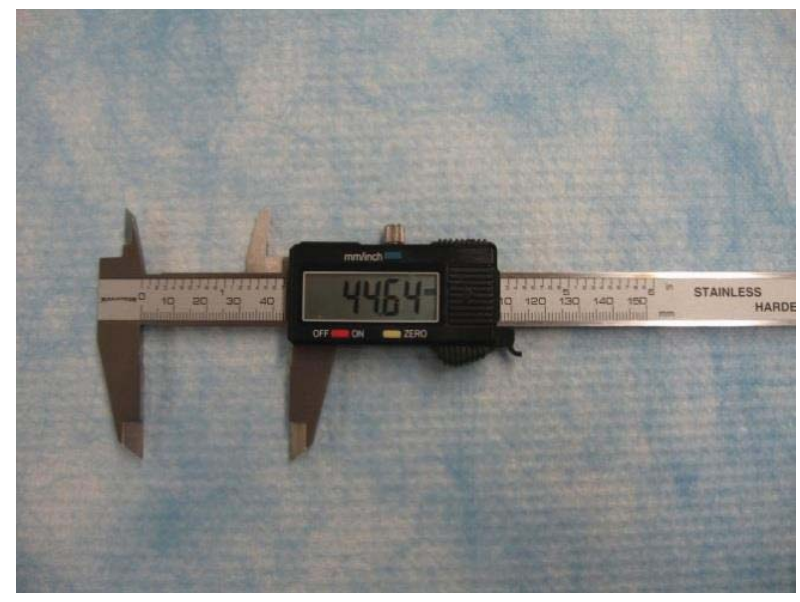

Figure 23: Digital Micrometer

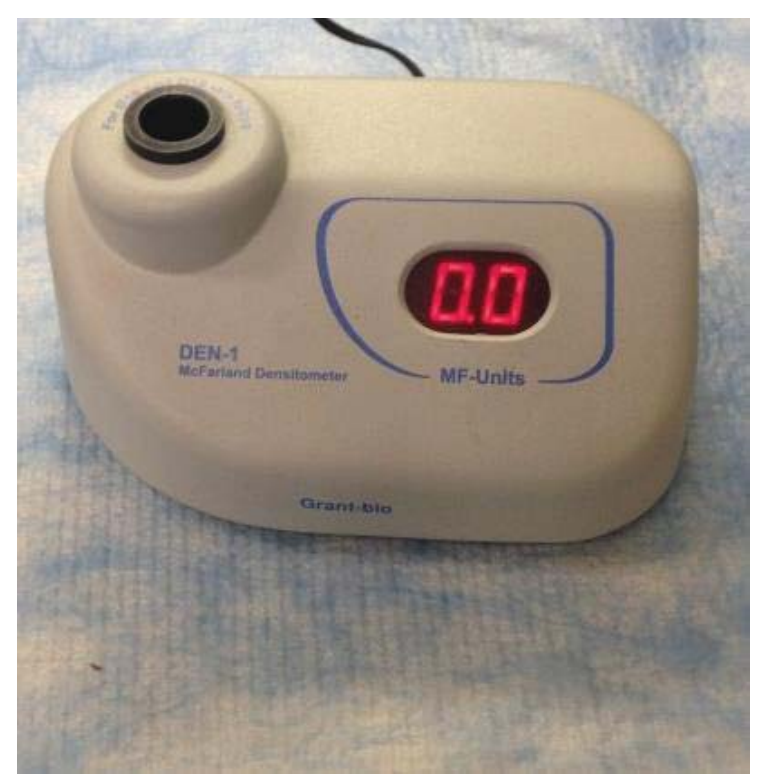

Figure 24: Light Spectrophotometer 
Figure 25: Illustration of five disc probiotic template on plates
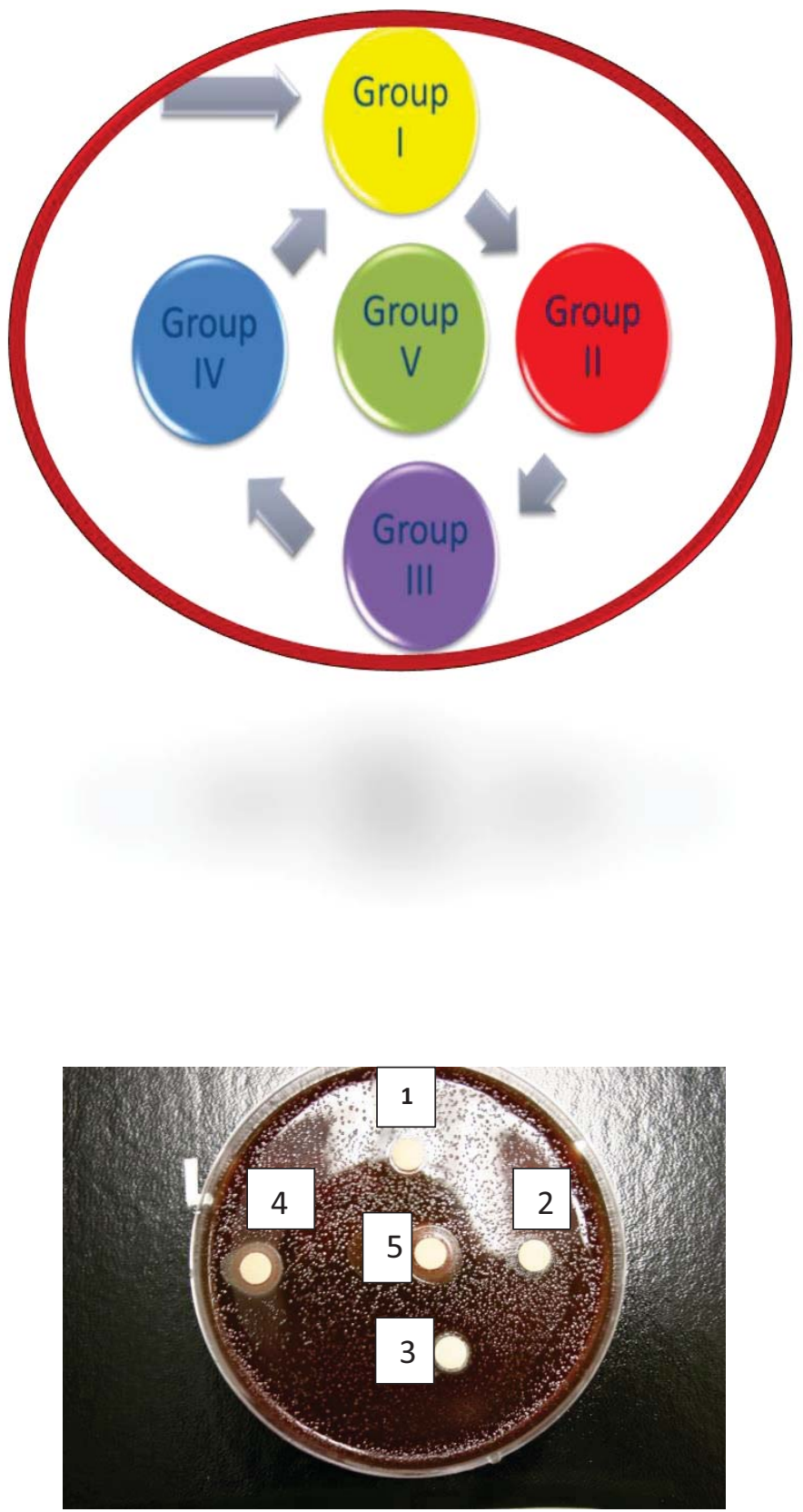

Figure 26: Five disc probiotic template on blood agar plate 


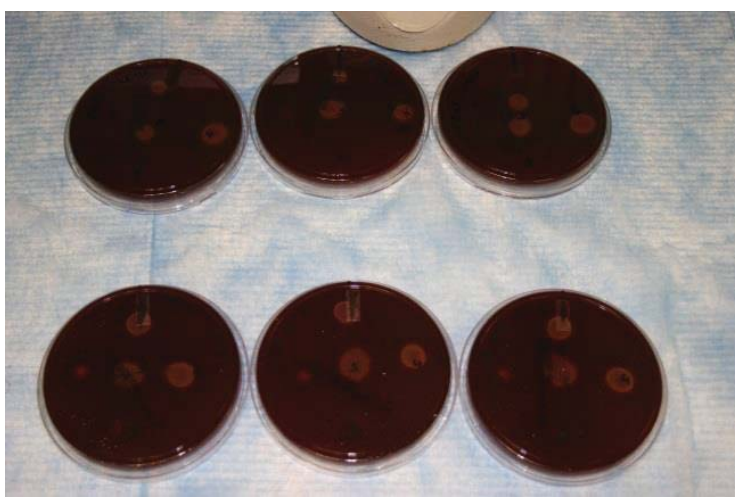

Figure 27: Six plates with five disc probiotic template on blood agar plates

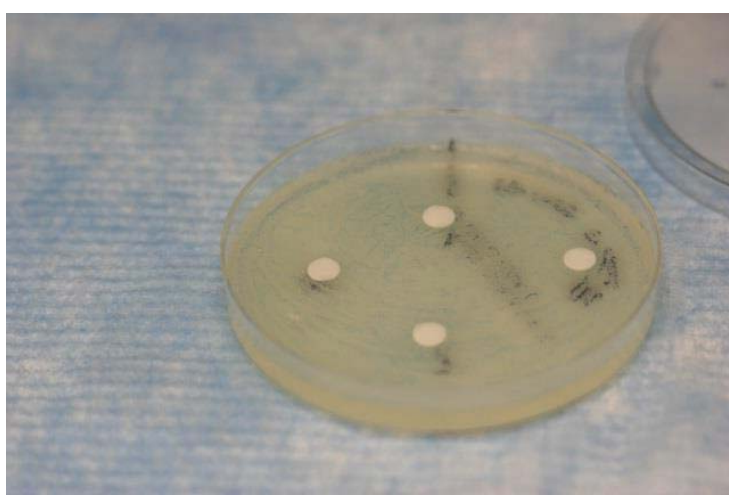

Figure 28: Control four disc template on Muller Hinton plate

\section{Phase 2: Biofilm stage testing; Intra-canal delivery vehicle for probiotics}

In this phase of the study, we evaluated biofilm staging by mixing pathogenic organisms with probiotic cocktails from individual groups, followed by serial dilutions and colony forming unit counts per ml. Only G I and G IV were evaluated for this part of the study, since they demonstrated the most significant zones of inhibition. G V was excluded from the study due to patent pending status of the probiotic cocktail.

Stage 1: Making of the Delivery Vehicle

Poloxamer F127 (407) was prepared by adding dry powder to the appropriate type and amount of broth (MRS and TSB were both utilized). Prebiotic mixtures (Inulin, 
Oligofructose , beta-glucan, larch arabinogalactan, glycerin and trechalose) were initially mixed and were considered to be included as part of the formulation, but due to time limitations of the study and to avoid having too many variables, it was decided to remove these prebiotics from the mixture as well as to prove the concept of probiotics outcompeting pathogenic organisms in the biofilm stage. This suggests that further studies be conducted with prebiotics added to the mixture and possibly enhancing the probiotic effect.

The poloxamer was dissolved in cold MRS broth at a concentration of $30 \%$ by a magnetic stirrer for 10 to 15 minutes until a homogenous mixture was obtained. The poloxamer was then sterilized and placed in the refrigerator at $4^{\circ} \mathrm{C}$ until testing was conducted. The MRS broth mixture was utilized for this study.

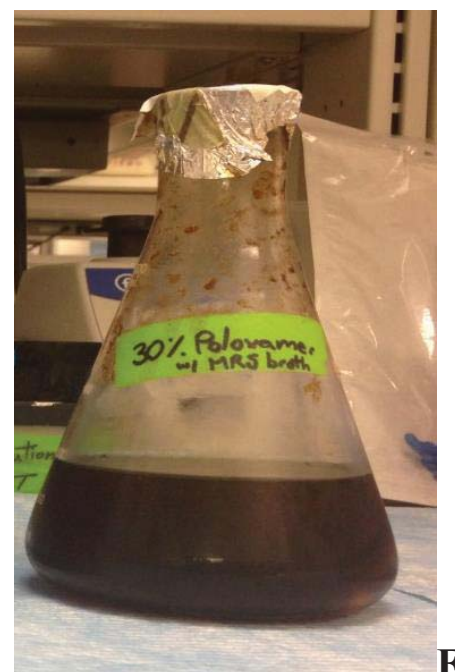

Figure 29: 30 \% Poloxamer 407 (Pluronic F-127) with MRS broth

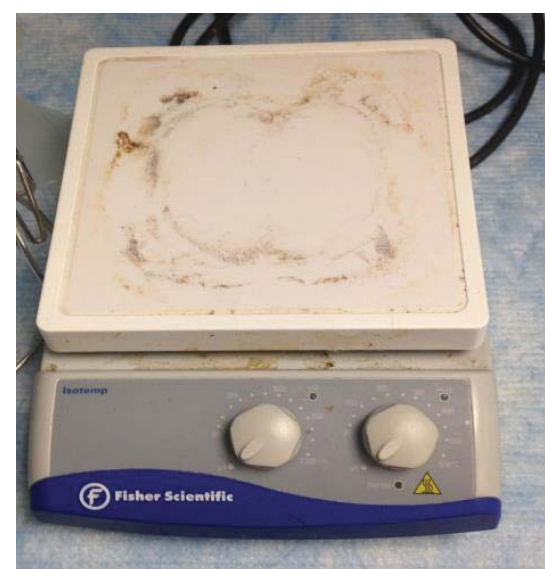

Figure 30: Magnetic Stirrer

Control testing: 
E. faecalis and C. albicans stocks were both prepared in TSB broth to a McFarland Standard of 1 via light spectrophotometry. A total of $9 \mathrm{mls}$ of poloxamer was placed in a test tube and $500 \mathrm{ml}$ of pathogenic organisms were added and vortexed at $4{ }^{\circ} \mathrm{C}$ in a refrigerated environment to allow homogenous mixture of poloxamer and microorganisms. Samples were then incubated for 48 hours in an incubator at $37^{\circ} \mathrm{C}$.

After 48 hours, serial dilutions of the pathogenic biofilm samples were prepared and plated on blood agar plates to evaluate colony forming units of the organisms. Serial dilutions were made by adding $0.1 \mathrm{ml}$ of poloxamer mix to $9.9 \mathrm{ml}$ sterile saline, followed by serially diluting the mixture by $0.1 \mathrm{ml}$ into $9.9 \mathrm{ml}$ sterile saline three times, reaching dilutions of $10^{-2}$, $10^{-4}, 10^{-6}$ respectively. Plating was conducted by adding $500 \mathrm{ml}$ of dilutions onto blood agar plates, followed by incubation at $37^{\circ} \mathrm{C}$ for 72 hours. CFU counts were evaluated.

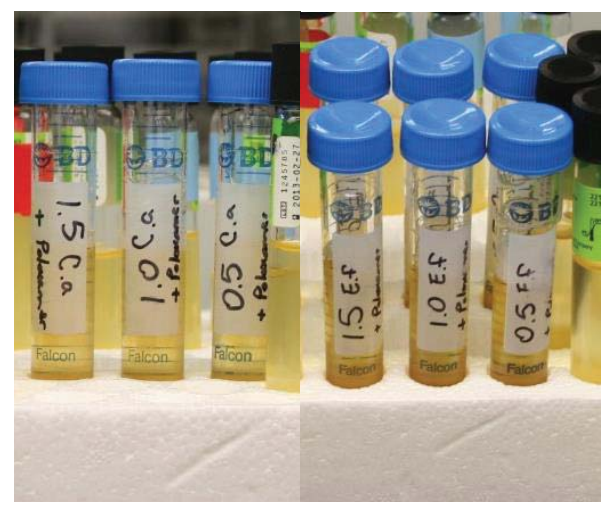

Figure 31: (A) and (B) Control E.f and C.a in poloxamer 407

(A)

(B)

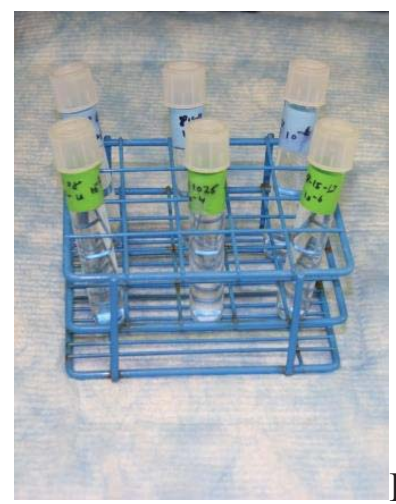

Figure 32: Serial Dilutions of $E$.faecalis and C.albicans 


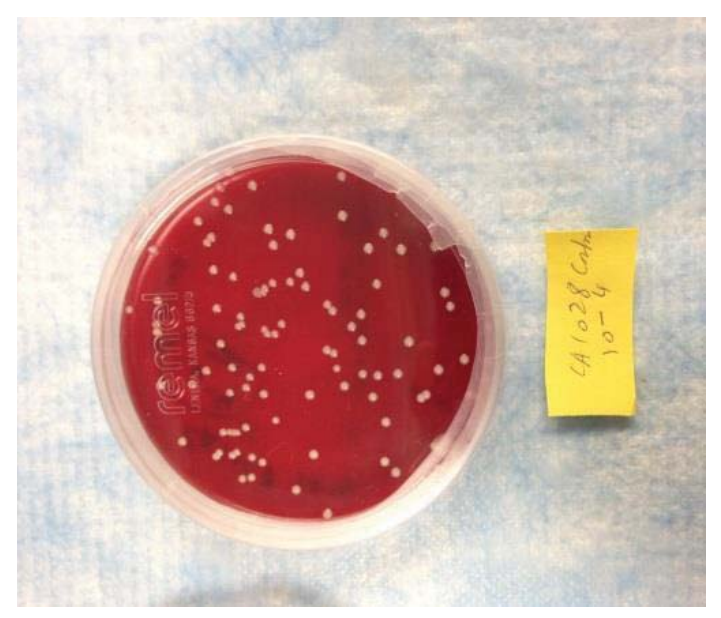

Figure 33: CFU/mL C.albicans $10^{-4}$ dilution

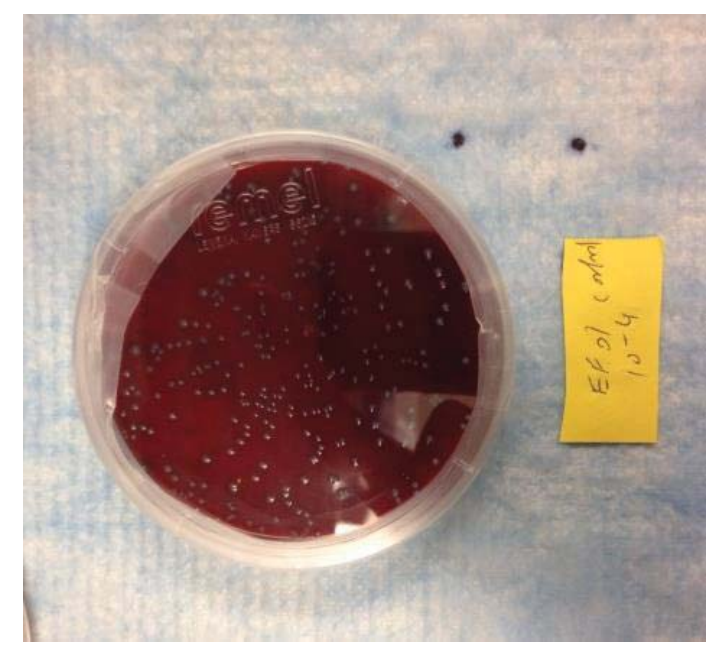

Figure 34: CFU/mL E.faecalis $10^{-4}$ dilution

\section{Testing for probiotic/pathogenic organism Poloxamer mixture:}

1. $9 \mathrm{ml}$ of the poloxamer mixture was placed in a test tube along with $500 \mathrm{ml}$ of $E$. faecalis at a $1 \mathrm{McF}$ arland standard. Following that, $500 \mathrm{ml}$ of test probiotic group 1 was added at 1.5 to 2 McFarland standards. 
2. $9 \mathrm{ml}$ of the poloxamer mixture was placed in a test tube and $500 \mathrm{ml}$ of E. faecalis was added at a $1 \mathrm{McF}$ arland standard. Following that, $500 \mathrm{ml}$ of test probiotic group 4 was added at 1.5 to 2 McFarland standards.

3. $9 \mathrm{ml}$ of the poloxamer mixture was placed in a test tube and $500 \mathrm{ml}$ of $C$ albicans at a 1 McFarland standard was added. Following that, $500 \mathrm{ml}$ of test probiotic group 4 was added at 1.5 to 2 McFarland standards.

4. $9 \mathrm{ml}$ of the poloxamer mixture was placed in a test tube and $500 \mathrm{ml}$ of C. albicans at a 1 McFarland standard was added. Following that, $500 \mathrm{ml}$ of test probiotic group 4 was added at 1.5 to 2 McFarland standards.

5. After 48 hours serial dilutions of the pathogenic biofilm samples were prepared and plated on blood agar plates to evaluate colony forming units of the organisms.

6. Serial dilutions were made by adding $0.1 \mathrm{ml}$ of poloxamer mix to $9.9 \mathrm{ml}$ sterile saline , followed by serially diluting the mixture by $0.1 \mathrm{ml}$ into $9.9 \mathrm{ml}$ sterile saline three times, reaching dilutions of $10^{-2}, 10^{-4}, 10^{-6}$ respectively.

7. Plating was conducted by adding $500 \mathrm{ml}$ of the dilutions onto blood agar plates followed by incubation at $37^{\circ} \mathrm{C}$ for 72 hours. CFU (colony forming units) were evaluated for all test groups and compared to controls based upon the dilutions that were performed to reflect the actual number of probiotics and pathogenic organisms in each group. 


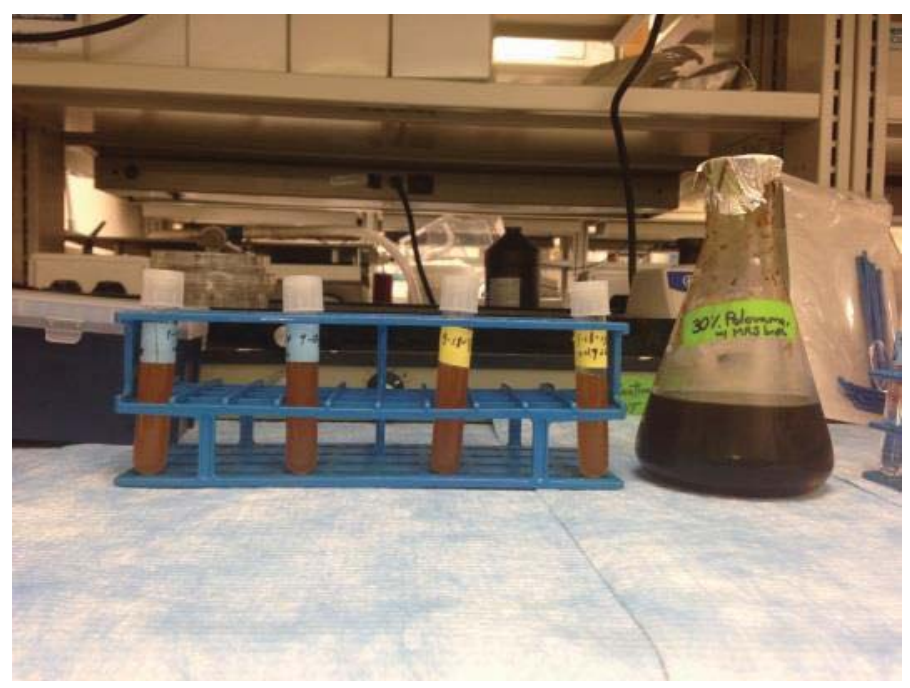

Figure 35: Poloxomer with pathogenic organisms and test probiotics 4 and 1 mixed together

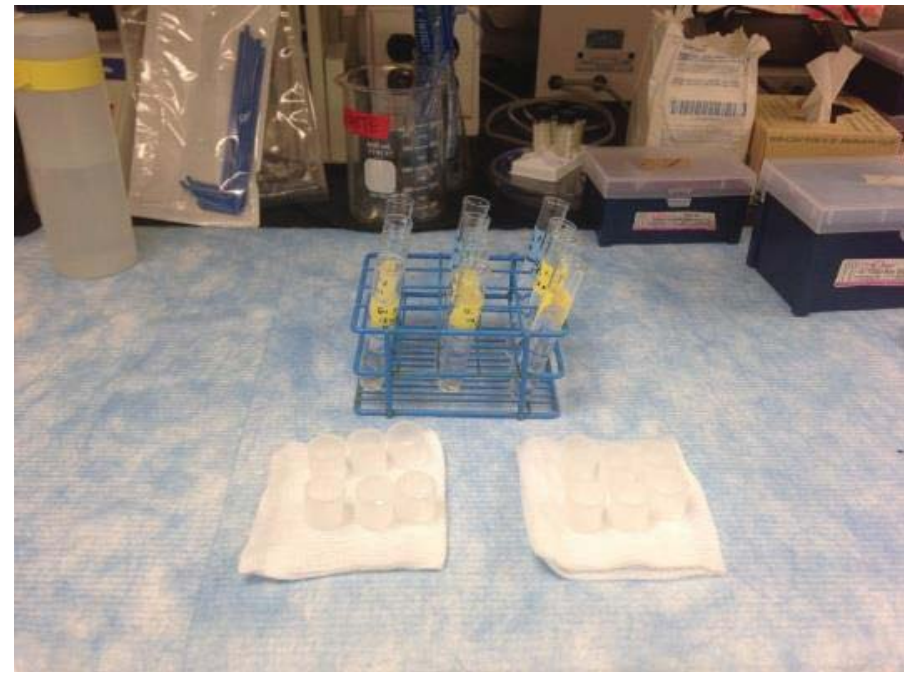

Figure 36: Serial dilutions of poloxamer/probiotic/pathogenic organisms mixed together. 


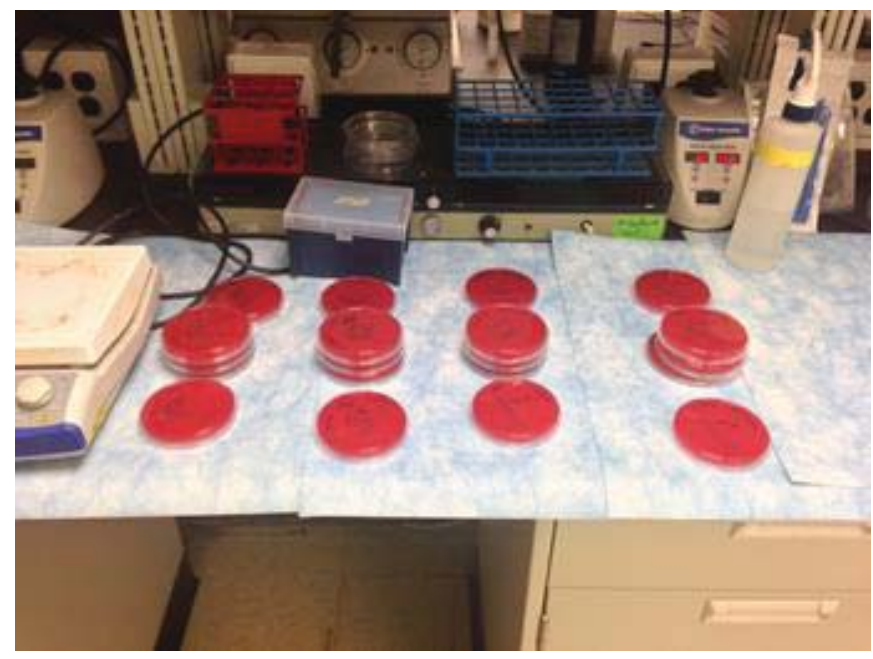

Figure 37: Serial dilutions of poloxamer/probiotic/pathogenic organisms mixed together on blood agar plates. 


\section{Chapter IV}

\section{$\underline{\text { Results }}$}

\section{Phase 1: ZOI}

Probiotics were screened for their antimicrobial/outcompeting activity against the selected microbes, E. faecalis and C. albicans.

The preliminary screening showed that G I, IV and V had antimicrobial activity against the specific pathogenic microbes tested. Clear zones of inhibition were seen around G I, IV and $\mathrm{V}$ with valid statistical significance $(\mathrm{P}<0.0001)$. Groups II and III showed variable results and therefore it could not be concluded that they are fully effective against the specific pathogenic organisms tested, and were subsequently eliminated from the biofilm stage testing. One observation in this study was that G I, IV and V all contained the common organisms $L$. acidophilus, L. casei, L. rhamnosus and B .longum in their groups, suggesting that further studies should focus on these particular organisms. Another observation was that prior to zones of inhibition being seen, a zone of growth was noted 24 hours after plating, which was then followed by the observation of zones of inhibition beginning at 48 hours. G III (Sachromyces boulardii) demonstrated a continuous ring or zone of growth around the disc with a small zone of inhibition against either of the pathogenic organisms. G IV and V showed the largest ZOI diameters. 
Zones were recorded via a digital micrometer and are noted in the table below (Table1)

1-Candida albicans Test:

- Table (1). ZOI Candida albicans 48Hours.

$\begin{array}{clllll}\text { Candida 48hrs } & \text { Group 1 } & \text { Group 2 } & \text { Group 3 } & \text { Group 4 } & \text { Group 5 } \\ \text { Test 1 } & 5.75 \mathrm{~mm} & 1.32 \mathrm{~mm} & 2.6 \mathrm{~mm} & 9.21 \mathrm{~mm} & 9.42 \mathrm{~mm} \\ \text { Test 2 } & 5.01 \mathrm{~mm} & 0.96 \mathrm{~mm} & 2.3 \mathrm{~mm} & 8.26 \mathrm{~mm} & 11.32 \mathrm{~mm} \\ \text { Test 3 } & 5.95 \mathrm{~mm} & 1.2 \mathrm{~mm} & 3.01 \mathrm{~mm} & 8.52 \mathrm{~mm} & 10.70 \mathrm{~mm}\end{array}$

ZOI at 48 hrs for group 1, 4 and 5 were statistically significant compared to groups 2 and 3 ( $\mathrm{P}<$ 0.0001) against $C$. albicans.

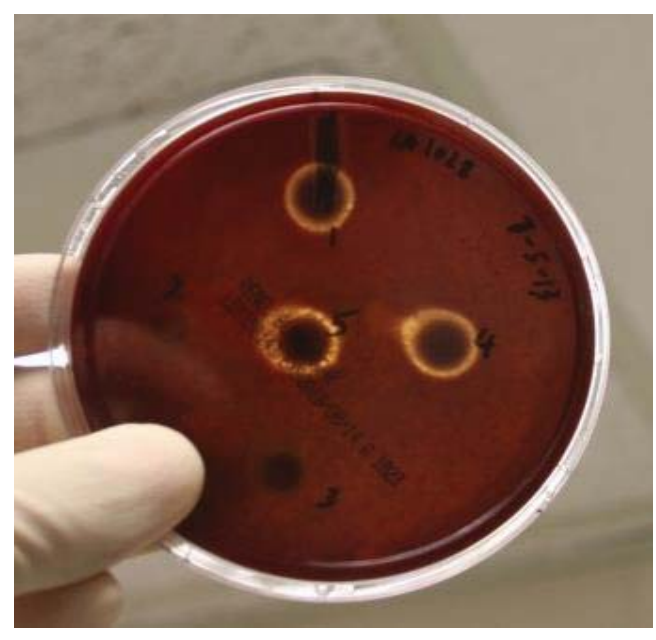

(A)

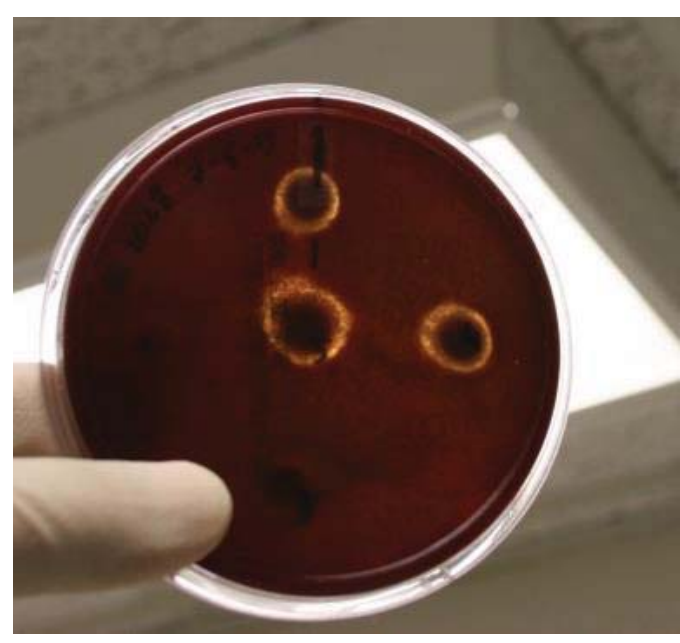

(B)

Figure 38: (A) and (B) ZOI's for Candida albicans 48-72hrs with five disc template on blood agar. 


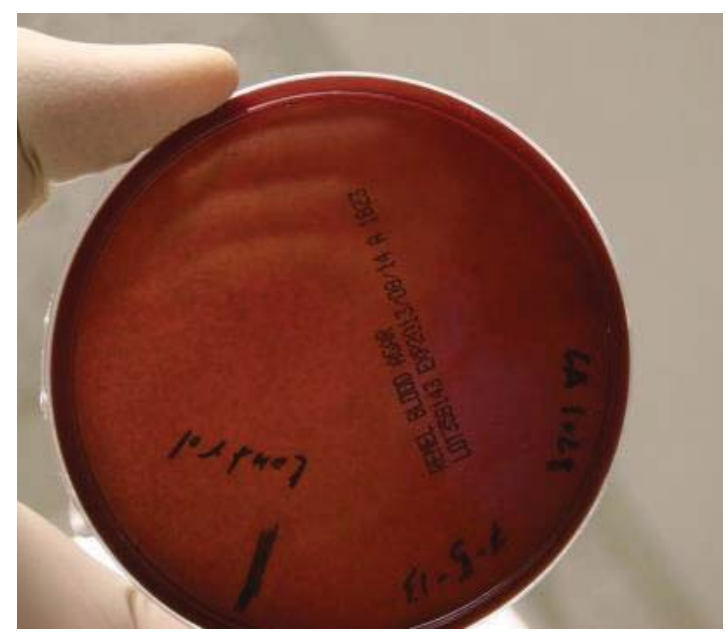

Figure 39 :Control Candida albicans on blood agar plate.

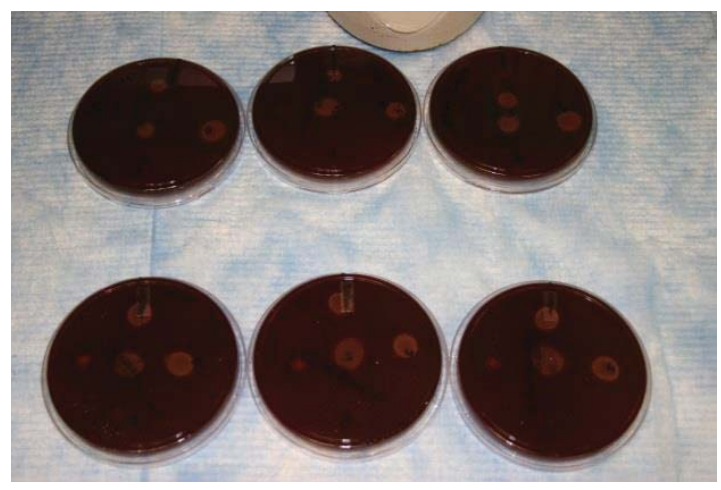

Figure 40: C.albicans and E.faecalis ZOI's (multiple plates).

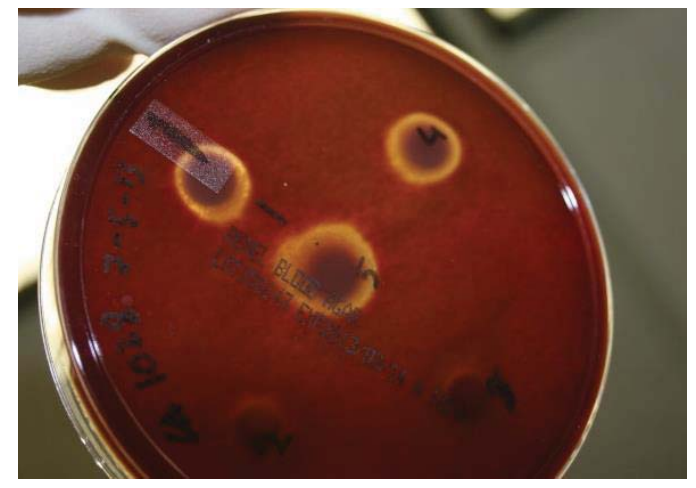

Figure 41: ZOI's for Candida albicans 1 week with five disc templates on blood agar. 


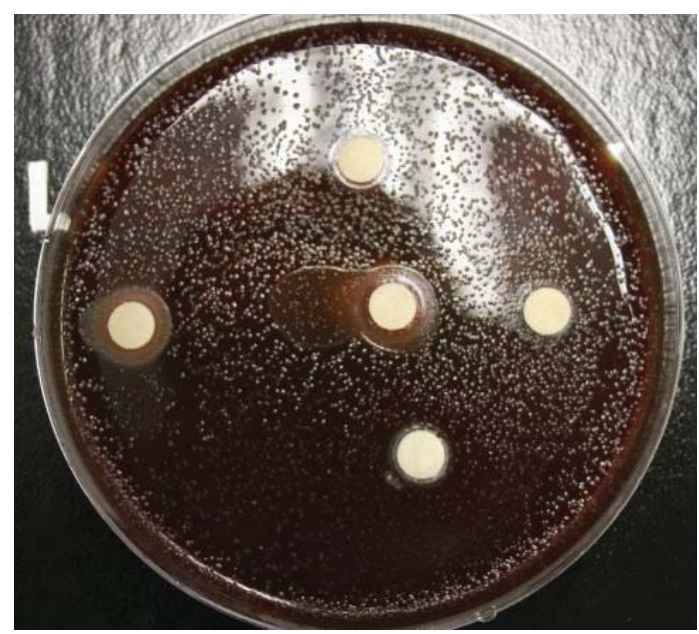

Figure 42: Zone of growth 24hrs for Candida albicans on blood agar.

- Table (2). ZOI Candida albicans 1 week.

$\begin{array}{clllll}\text { Candida } & \text { Group 1 } & \text { Group 2 } & \text { Group 3 } & \text { Group 4 } & \text { Group 5 } \\ 1 \text { week } & & & & & \\ \text { Test 1 } & 7.05 \mathrm{~mm} & 2.1 \mathrm{~mm} & 3.21 \mathrm{~mm} & 9.94 \mathrm{~mm} & 10.79 \mathrm{~mm} \\ \text { Test 2 } & 7.24 \mathrm{~mm} & 2.13 \mathrm{~mm} & 3.26 \mathrm{~mm} & 9.82 \mathrm{~mm} & 12.64 \mathrm{~mm} \\ \text { Test 3 } & 7.95 \mathrm{~mm} & 1.89 \mathrm{~mm} & 3.3 \mathrm{~mm} & 10.4 \mathrm{~mm} & 12.53 \mathrm{~mm} \\ & & & & & \end{array}$

ZOI at 1 week for group 1, 4 and 5 were statistically significant compared to groups 2 and 3 (P< 0.0001 ) against $C$. albicans.

Statistical analysis was conducted by least squares means and one way ANOVA for comparing all groups. 


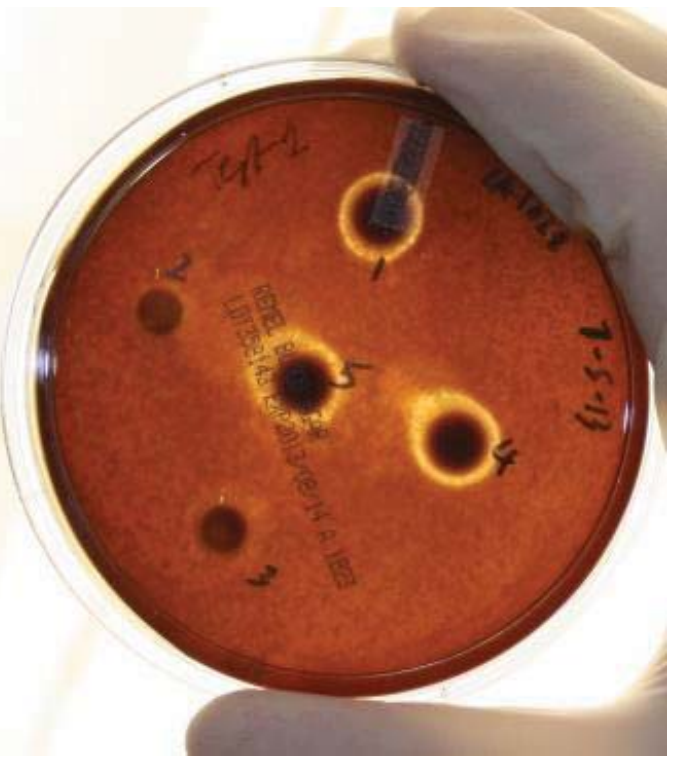

(A)

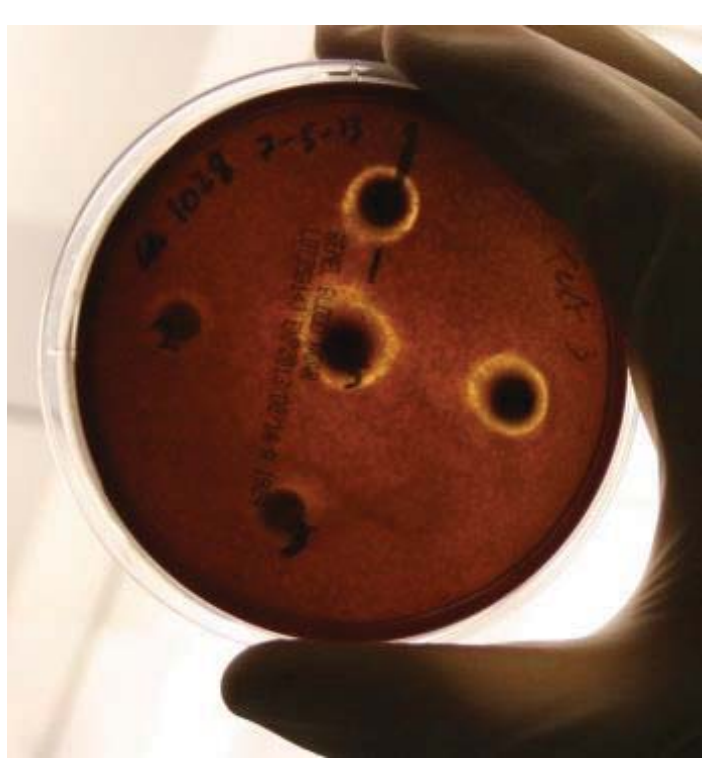

(B)

Figure 43: (A) and (B) ZOI's for Candida albicans one week with five disc template on blood agar.

- Whole Model for Candida albicans:48hrs and 1 week

- Table ( 3 ) .Analysis of variance (ANOVA) for Zone of Inh by Group =CA

\begin{tabular}{l|r|r|r|r|r|}
\hline \multicolumn{1}{|c|}{ Analysis of Variance } \\
\hline Source & DF & $\begin{array}{r}\text { Sum of } \\
\text { Squares }\end{array}$ & Mean Square & F Ratio \\
\hline Model & 9 & 415.49452 & 46.1661 & 153.8561 \\
\hline Error & 20 & 6.00120 & 0.3001 & Prob $>$ F \\
\hline $\begin{array}{l}\text { C. } \\
\text { Total }\end{array}$ & 29 & 421.49572 & & $<.0001^{*}$ \\
\hline
\end{tabular}

- Table (4). Effect Tests for Zone of Inh by Group =CA 


\begin{tabular}{|c|c|c|c|c|c|}
\hline \multicolumn{6}{|l|}{ Effect Tests } \\
\hline Source & Nparm & $\overline{D F}$ & $\begin{array}{r}\text { Sum of } \\
\text { Squares }\end{array}$ & F Ratio & $\begin{array}{r}\text { Prob }> \\
F\end{array}$ \\
\hline time & 1 & 1 & 11.68128 & 38.9298 & $<.0001 *$ \\
\hline Group & 4 & 4 & 402.35629 & 335.2299 & $<.0001^{*}$ \\
\hline time*Group & 4 & 4 & 1.45695 & 1.2139 & 0.3361 \\
\hline
\end{tabular}

- Table (5).Least Squares Means Whole Model=CA

\begin{tabular}{|l|r|r|r|r|}
\hline Level & $\begin{array}{r}\text { Least Sq } \\
\text { Mean }\end{array}$ & & Std Error & Mean \\
\hline $\begin{array}{l}\text { Group } \\
1\end{array}$ & 6.491667 & & 0.22362916 & 6.4917 \\
\hline $\begin{array}{l}\text { Group } \\
2\end{array}$ & 1.600000 & 0.22362916 & 1.6000 \\
\hline $\begin{array}{l}\text { Group } \\
3\end{array}$ & 2.946667 & 0.22362916 & 2.9467 \\
\hline $\begin{array}{l}\text { Group } \\
4\end{array}$ & 9.358333 & 0.22362916 & 9.3583 \\
\hline $\begin{array}{l}\text { Group } \\
5\end{array}$ & 11.233333 & 0.22362916 & 11.2333 \\
\hline \\
time*Group
\end{tabular}

- $\quad$ Table (6) .Least Squares Means by Day=CA

\begin{tabular}{|c|c|c|}
\hline \multicolumn{3}{|c|}{ Least Squares Means Table } \\
\hline Level & $\begin{array}{r}\text { Least Sq } \\
\text { Mean }\end{array}$ & Std Error \\
\hline 2 days, Group 1 & 5.570000 & 0.31625939 \\
\hline 2 days, Group 2 & 1.160000 & 0.31625939 \\
\hline 2 days, Group 3 & 2.636667 & 0.31625939 \\
\hline 2 days, Group 4 & 8.663333 & 0.31625939 \\
\hline 2 days, Group 5 & 10.480000 & 0.31625939 \\
\hline 7 days, Group 1 & 7.413333 & 0.31625939 \\
\hline 7 days, Group 2 & 2.040000 & 0.31625939 \\
\hline 7 days, Group 3 & 3.256667 & 0.31625939 \\
\hline 7 days, Group 4 & 10.053333 & 0.31625939 \\
\hline 7 days, Group 5 & 11.986667 & 0.31625939 \\
\hline
\end{tabular}


- Figure 44: LS Means Plot Candida albicans

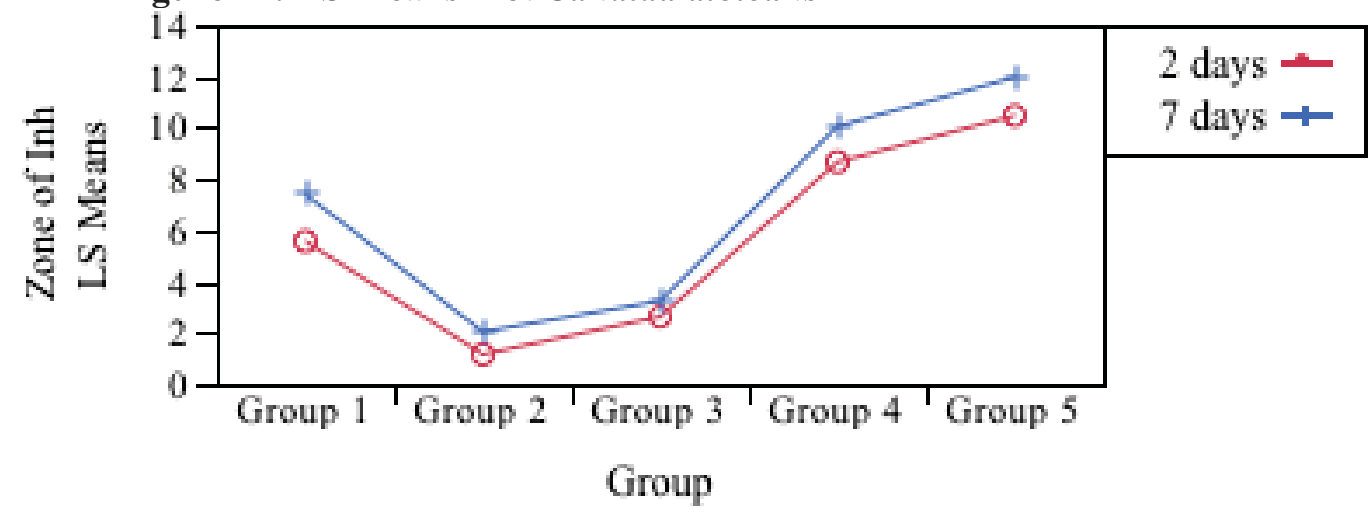

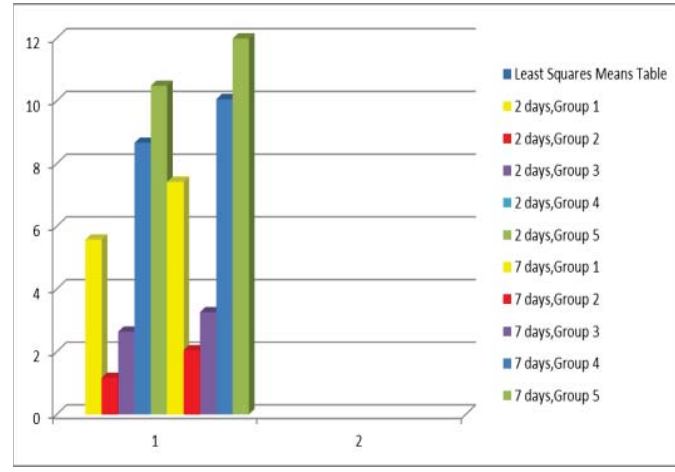

(A)

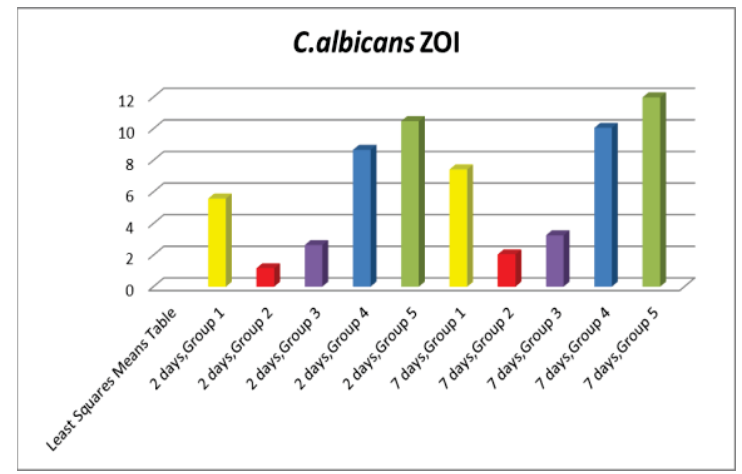

(B)

Figure 45: (A) and (B) LS Means bar Graph $C$.albicans 


\section{2-E.faecalis test:}

- Table (7). ZOI Enterococci faecalis 48Hours.

$\begin{array}{llllll}\text { E.faecalis } & \text { Group 1 } & \text { Group 2 } & \text { Group 3 } & \text { Group 4 } & \text { Group5 } \\ 48 \text { Hrs } & & & & \\ \text { Test 1 } & 5.08 \mathrm{~mm} & 2.8 \mathrm{~mm} & 4.97 \mathrm{~mm} & 8.96 \mathrm{~mm} & 10.23 \mathrm{~mm} \\ \text { Test 2 } & 5.36 \mathrm{~mm} & 1.56 \mathrm{~mm} & 4.03 \mathrm{~mm} & 9.84 \mathrm{~mm} & 9.55 \mathrm{~mm} \\ \text { Test 3 } & 6.04 \mathrm{~mm} & 2.09 \mathrm{~mm} & 3.6 \mathrm{~mm} & 10.13 \mathrm{~mm} & 9.9 \mathrm{~mm}\end{array}$

ZOI at 48 hrs for group 1, 4 and 5 were statistically significant compared to groups 2 and 3 (P< 0.0001) against $E$. faecalis.

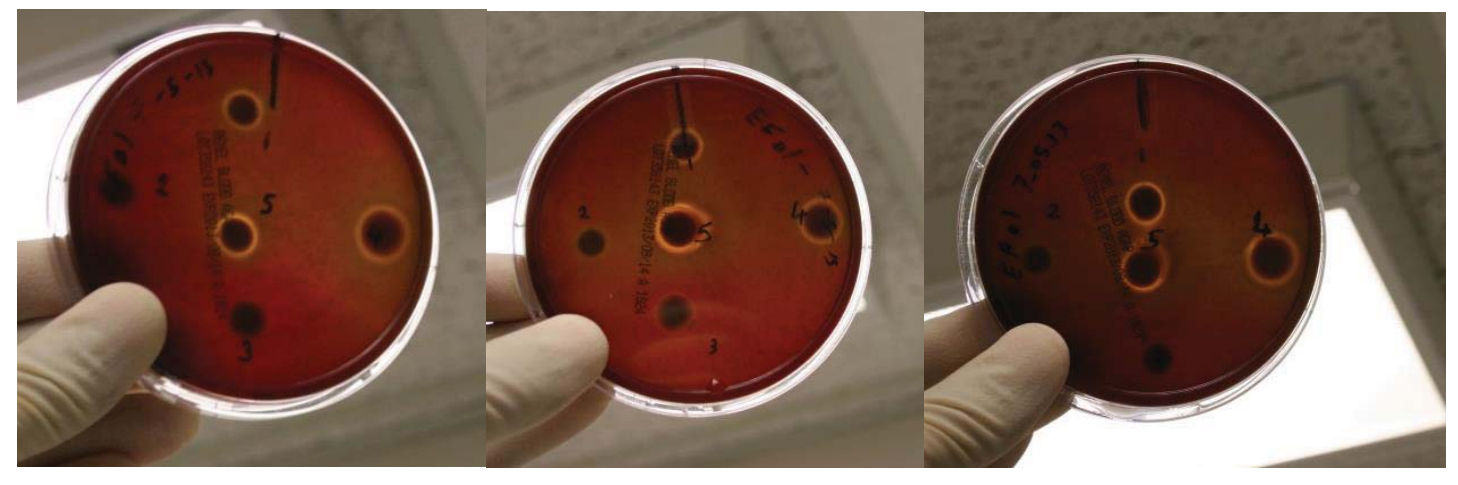

(A)

(B)

(C)

Figure 46: (A), (B) and (C) ZOI's for Enterococci faecalis 48-72hrs with five disc templates on blood agar. 


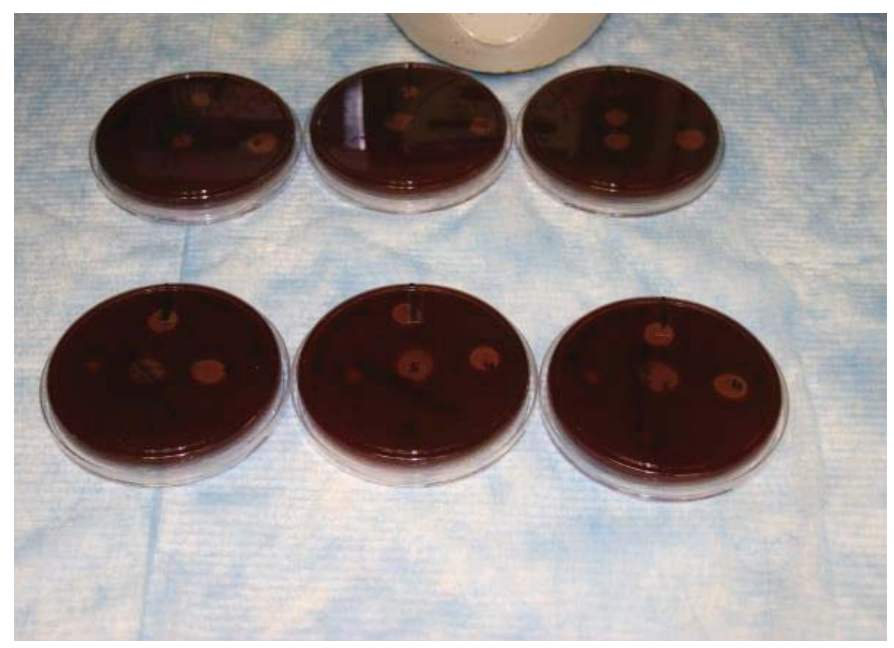

Figure 47: E.faecalis and C.albicans ZOI's (multiple plates).

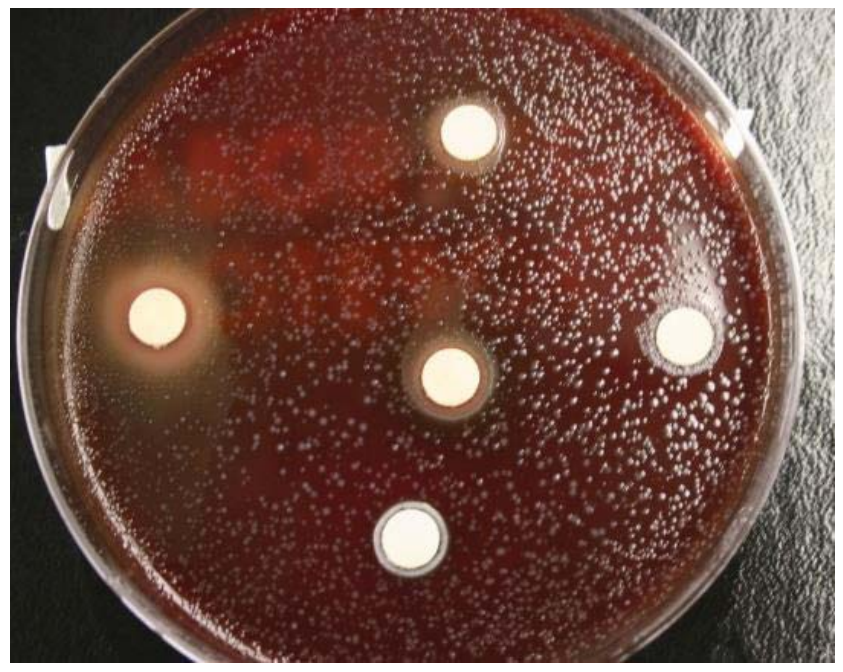

Figure 48: Zone of growth 24 hrs for Enterococci faecalis on blood agar. 


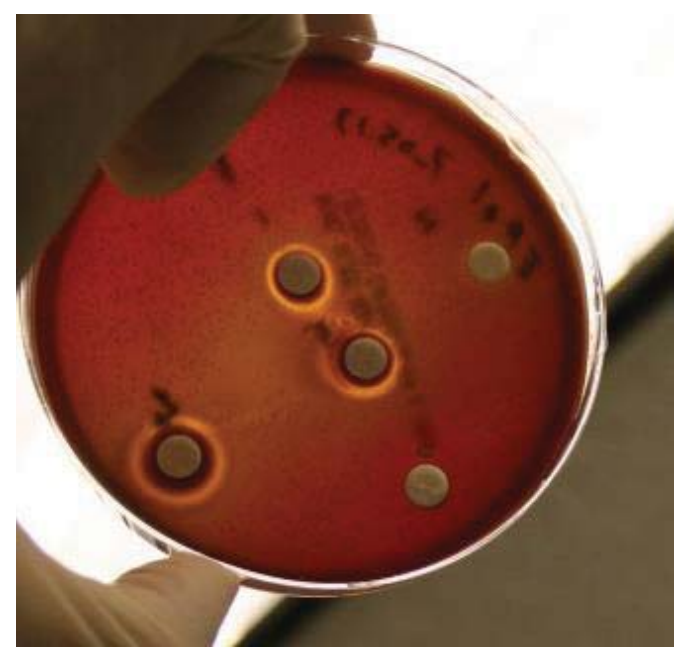

(A)

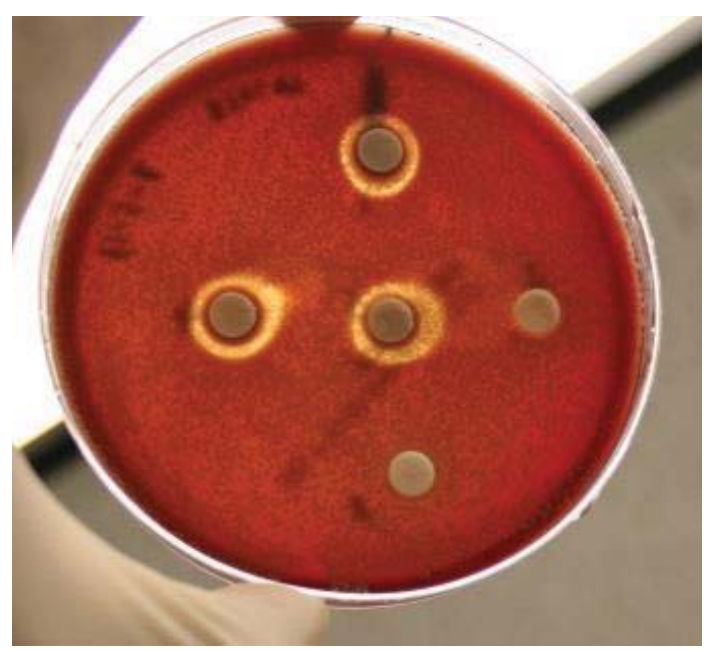

(B)

Figure 49: (A) and (B) ZOI's for Enterococci faecalis 48-72hrs with five disc template on blood agar.

- Table (8). ZOI Enterococci faecalis 1 week.

\begin{tabular}{llllll}
\hline E.faecalis & Group 1 & Group 2 & Group 3 & Group 4 & Group 5 \\
1 week & & & & & \\
Test 1 & $6.01 \mathrm{~mm}$ & $4.61 \mathrm{~mm}$ & $5.3 \mathrm{~mm}$ & $9.9 \mathrm{~mm}$ & $12.8 \mathrm{~mm}$ \\
Test 2 & $6.2 \mathrm{~mm}$ & $4.47 \mathrm{~mm}$ & $5.4 \mathrm{~mm}$ & $11.92 \mathrm{~mm}$ & $12.2 \mathrm{~mm}$ \\
Test 3 & $7.9 \mathrm{~mm}$ & $3.89 \mathrm{~mm}$ & $4.2 \mathrm{~mm}$ & $11.6 \mathrm{~mm}$ & $12.72 \mathrm{~mm}$ \\
\end{tabular}

ZOI at 1 week for group 1, 4 and 5 were statistically significant compared to groups 2 and 3 ( $\mathrm{P}<$ 0.0001 ) against $E$. faecalis. 


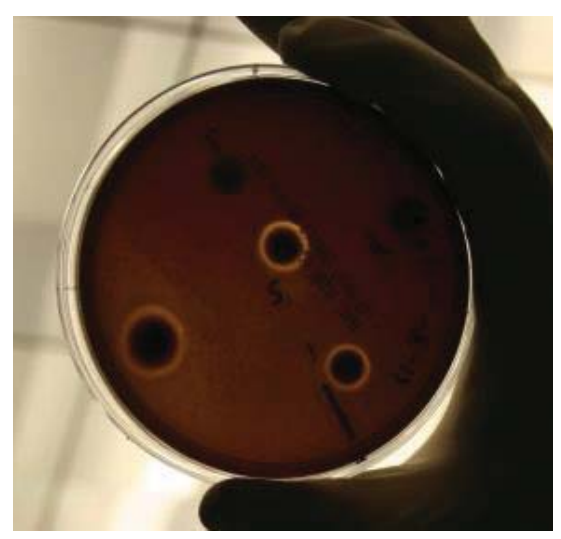

(A)

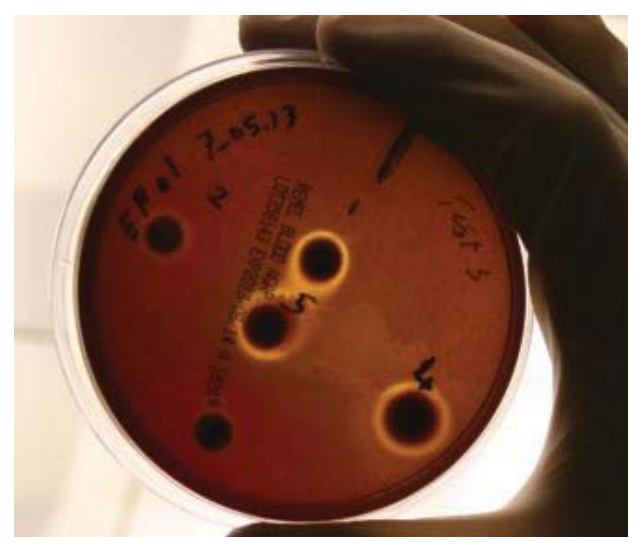

(C)

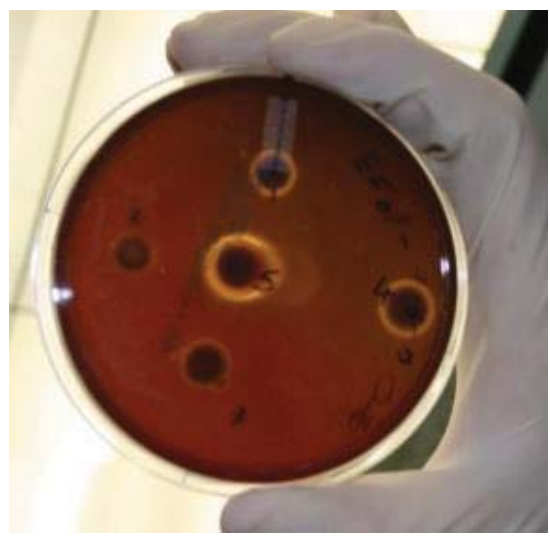

(B)

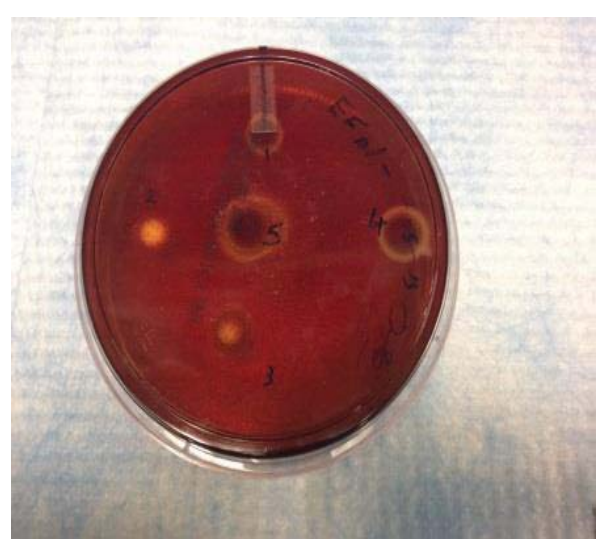

(D)

Figure 50: (A), (B), (C) and (D) ZOI's for Enterocci faecalis one week with five disc template on blood agar. 
Statistical analysis was conducted by least squares means and one way ANOVA for comparing all groups.

- Whole Model for Enterococci faecalis:48hrs and 1 week

- Table (9). Analysis of variance (ANOVA) for Zone of Inh by Group =EF

\begin{tabular}{|l|c|l|c|c|}
\hline Analysis of Variance \\
\hline Source & DF & Sum of Squares & Mean Square & F Ratio \\
\hline Model & 9 & 325.38508 & 36.1539 & 79.1870 \\
\hline Error & 20 & 9.13127 & 0.4566 & Prob $>$ F \\
\hline C. Total & 29 & 334.51635 & & $<.0001^{*}$ \\
\hline
\end{tabular}

- Table (10). Effect Tests for Zone of Inh by Group =EF

\section{Effect Tests}

\begin{tabular}{l|r|r|r|r|r|r}
\hline Source & Nparm & DF & $\begin{array}{r}\text { Sum of } \\
\text { Squares }\end{array}$ & F Ratio & Prob > F & \\
\hline time & 1 & 1 & 20.80001 & 45.5578 & $<.0001^{*}$ & \\
\hline Group & 4 & 4 & 301.08858 & 164.8668 & $<.0001^{*}$ & \\
\hline time*Group & 4 & 4 & 3.49649 & 1.9146 & 0.1473 & \\
\hline
\end{tabular}


- Table(11) .Least Squares Means Whole Model=EF

Least Squares Means Table
\begin{tabular}{|l|r|r|r|}
\hline Level & $\begin{array}{r}\text { Least Sq } \\
\text { Mean }\end{array}$ & Std Error & Mean \\
\hline $\begin{array}{l}\text { Group } \\
1\end{array}$ & 6.098333 & 0.27585121 & 6.0983 \\
\hline $\begin{array}{l}\text { Group } \\
2\end{array}$ & 3.236667 & 0.27585121 & 3.2367 \\
\hline $\begin{array}{l}\text { Group } \\
3\end{array}$ & 4.583333 & 0.27585121 & 4.5833 \\
\hline $\begin{array}{l}\text { Group } \\
4\end{array}$ & 10.391667 & 0.27585121 & 10.3917 \\
\hline $\begin{array}{l}\text { Group } \\
5\end{array}$ & 11.233333 & 0.27585121 & 11.2333 \\
\hline
\end{tabular}

Time*Group

Table (12). Least Squares Means by Day $=\mathrm{EF}$

\begin{tabular}{|l|r|r|}
\hline Level & $\begin{array}{r}\text { Least Sq } \\
\text { Mean }\end{array}$ & Std Error \\
\hline 2 days, Group 1 & 5.493333 & 0.39011252 \\
\hline 2 days, Group 2 & 2.150000 & 0.39011252 \\
\hline 2 days, Group 3 & 4.200000 & 0.39011252 \\
\hline 2 days, Group 4 & 9.643333 & 0.39011252 \\
\hline 2 days, Group 5 & 9.893333 & 0.39011252 \\
\hline 7 days, Group 1 & 6.703333 & 0.39011252 \\
\hline 7 days, Group 2 & 4.323333 & 0.39011252 \\
\hline 7 days, Group 3 & 4.966667 & 0.39011252 \\
\hline 7 days, Group 4 & 11.140000 & 0.39011252 \\
\hline 7 days, Group 5 & 12.573333 & 0.39011252 \\
\hline
\end{tabular}




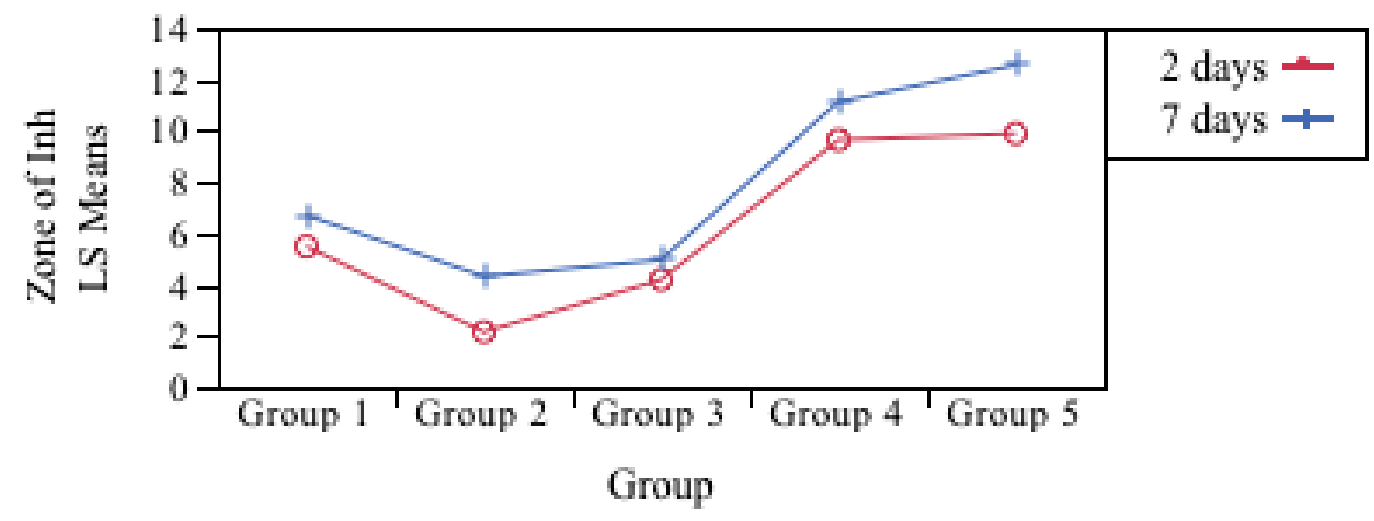

Figure (51): LS Means plot E. faecalis

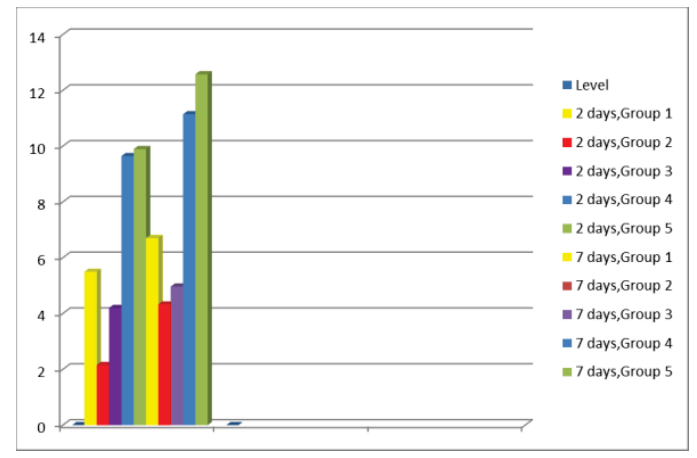

(A)

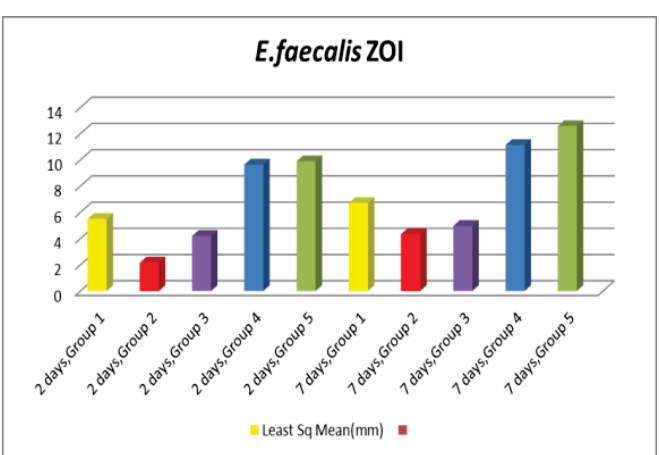

(B)

Figure 52: (A) and (B) LS Means bar Graph E. faecalis

Based on the statistical analysis, G I, IV and V were all effective in inhibiting and/or outcompeting endodontic pathogenic organisms E. faecalis and C. albicans showing a tangible ZOI $(\mathrm{P}<0.0001)$. 


\section{Phase 2: Biofilm staging and CFU counts}

Colony forming unit counts were conducted manually by dividing the blood agar plates into four quadrants and counting the colonies in each segment with a click counter and a pen. Controls of E. faecalis and C. albicans in the poloxamer mixture were plated after serial dilutions of $10^{-2}, 10^{-4}$, and $10^{-6}$. Dilutions of $\left(10^{-2}\right)$ both pathogenic organisms showed colonies which were too numerous to count $\left(10^{-4}\right)$. Dilutions of $\left(10^{-4)}\right.$ showed $7.5 \times 10^{5}(75)$ colonies for C. albicans, and $1.75 \times 10^{6}(175)$ colonies for E. faecalis. $\left(10^{-6}\right)$ dilutions of $\left(10^{-6)}\right.$ showed 3 colonies for C. albicans and 7 colonies for E. faecalis.

After CFU count was conducted for the controls, test probiotic groups I and IV were mixed with the pathogenic organisms in the poloxamer mixture, plated and counted. Colonies of the probiotics were less, compared to colonies of the controls. Due to financial limitations of the study, PCR (DNA or RNA sequencing) testing was not conducted, but random sampling by a sterile loop was taken from the colonies on the blood agar test plate, followed by gram staining and observation under a light microscope for evaluation of type of organisms present. None of the probiotic groups had yeast or cocci; therefore any observations of yeast or cocci during the random sampling would have indicated incomplete elimination of either of the pathogenic organisms.

Multiple random samples were taken from the plates. No cocci were found in the random samples, indicating that $E$. faecalis was completely eliminated in the biofilm stage when mixed with either groups I or IV.

Yeast ( $C$. albicans) colonies were found in the samples, but were limited in colony numbers and were easily distinguished from probiotics due to the size of the yeast colony. Results for the probiotic/pathogenic organism poloxamer mixture were as follows: Yeast (C. albicans) colonies were found in the samples but were limited in colony numbers and were easily distinguished from probiotics due to the size of the yeast colony. Results for the probiotic /pathogenic organism poloxamer mixture were as follows: 


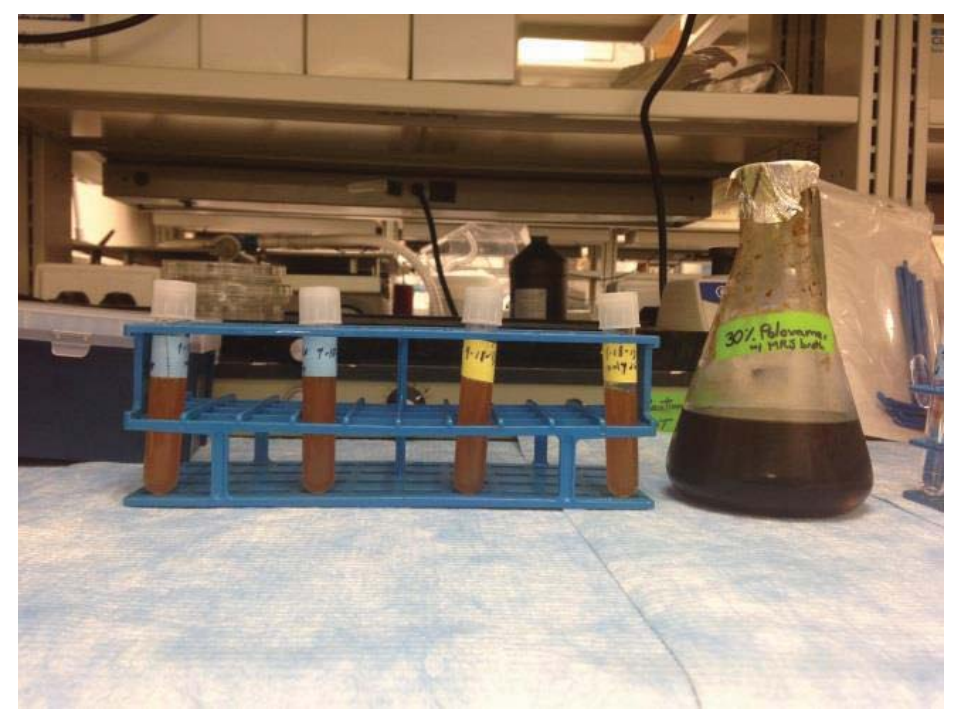

Figure 53: Poloxomer with pathogenic organisms and test probiotics 4 and 1 mixed together

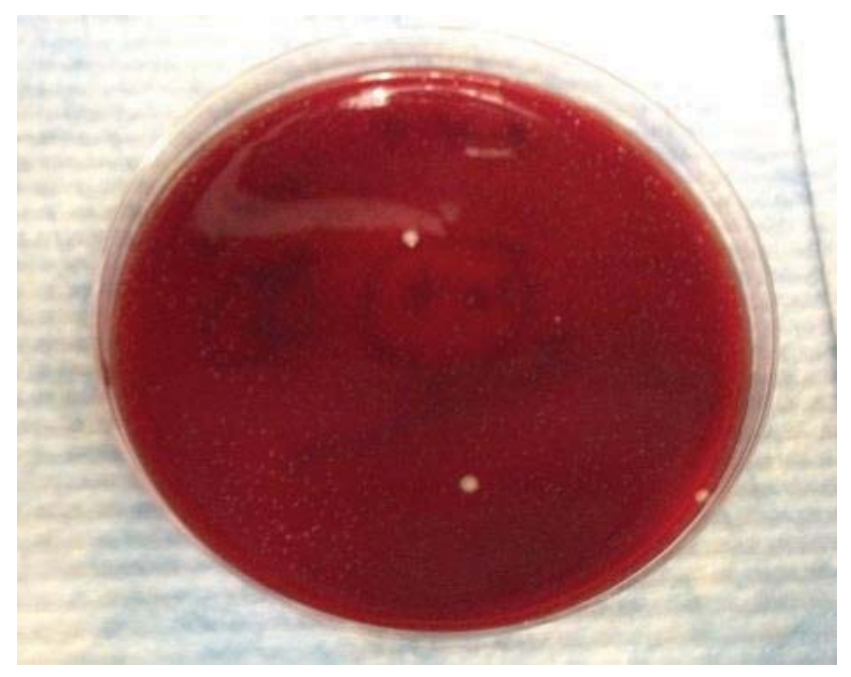

Figure 54: CFU/mL Group I and C.albicans $10^{-4}$ dilution -72hrs 


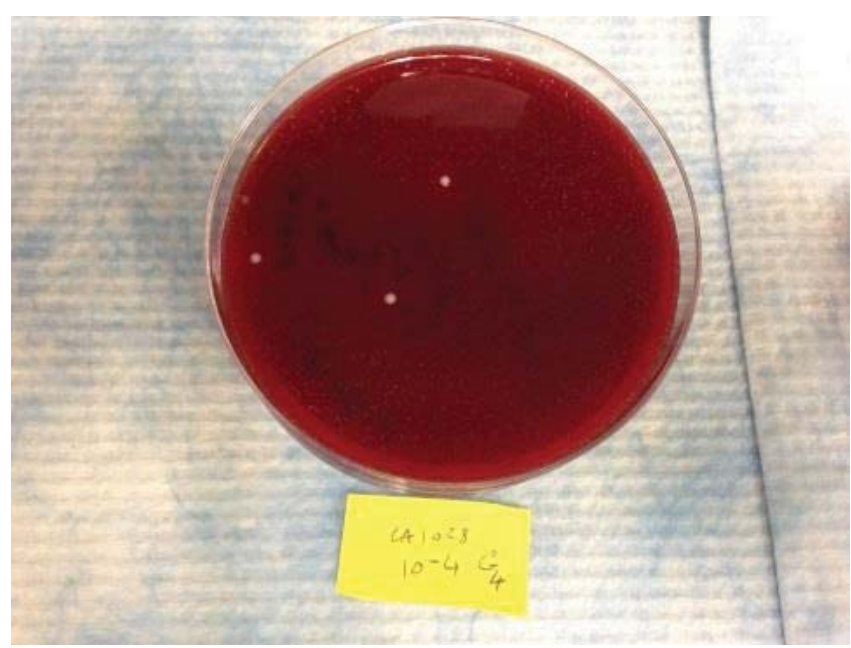

Figure 55: CFU/mL Group IV and C.albicans $10^{-4}$ dilution $-72 \mathrm{hrs}$

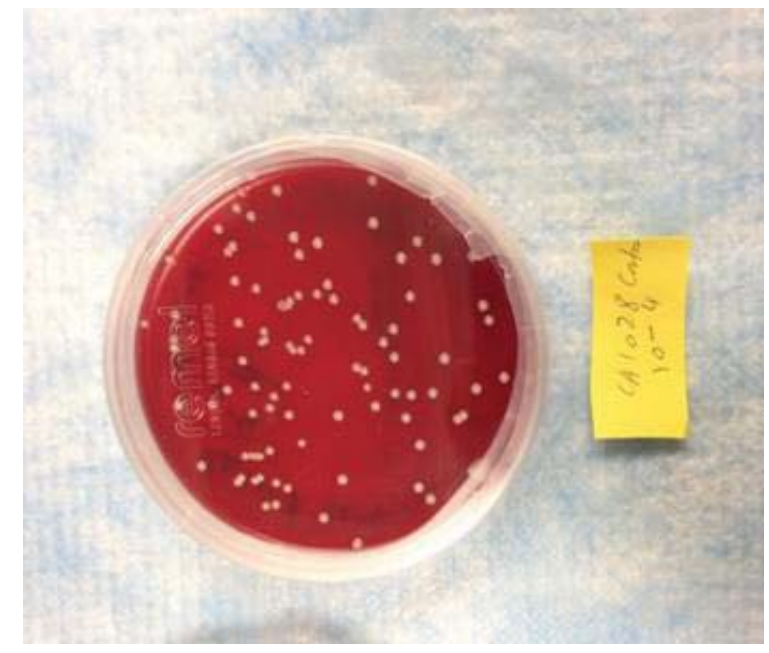

Figure 56: CFU/mL C.albicans $10^{-4}$ dilution (Control) -72hrs 


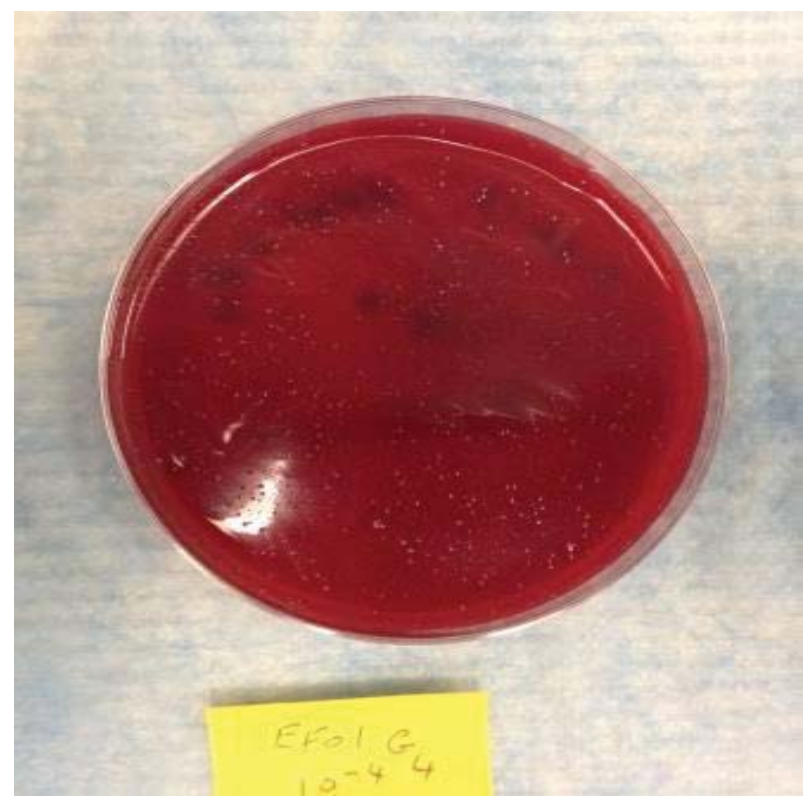

Figure 57: CFU/mL Group IV and E.faecalis $10^{-4}$ dilution $-72 \mathrm{hrs}$

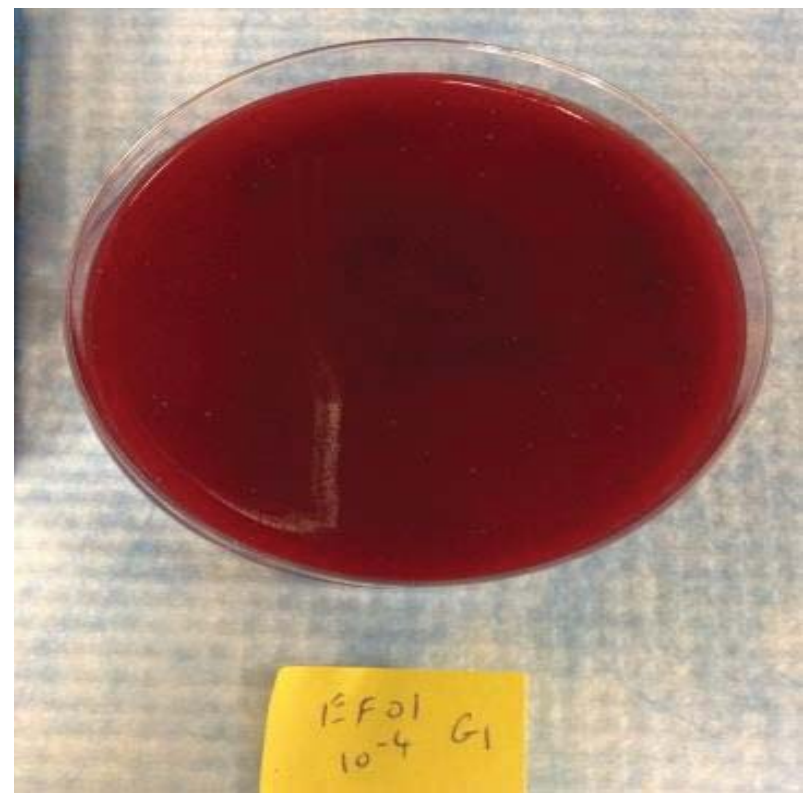

Figure 58: CFU/mL Group 4 and E.faecalis $10^{-4}$ dilution $-72 \mathrm{hrs}$ 


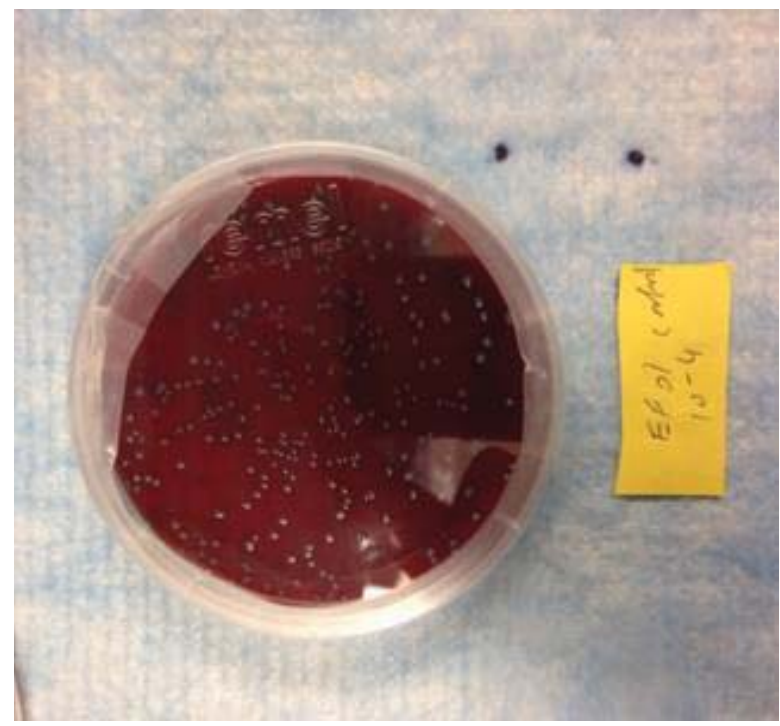

Figure 59: CFU/mL E.faecalis $10^{-4}$ dilution (Control) $-72 \mathrm{hrs}$

\section{Poloxamer results (Biofilm stage testing):}

- Table (13). CFU/mL Candida albicans -72hrs

\begin{tabular}{|l|c|c|c|}
\hline Test organism & $\begin{array}{c}\text { Group 1 test } \\
\text { (Candida and Group 1) }\end{array}$ & $\begin{array}{c}\text { Group 4 test } \\
\text { (Candida and Group 4) }\end{array}$ & Control (Candida alone) \\
\hline Candida colonies & $\begin{array}{c}4.0 \times 10^{3} \\
\text { (2 log difference) }\end{array}$ & $\begin{array}{c}1.5 \times 10^{4} \\
(1 \text { log difference) }\end{array}$ & $7.5 \times 10^{5}$ \\
\hline
\end{tabular}


Figure 60: CFU/ml For Candida albicans (control), Group 1 and Group 4 mixed with Poloxamer-72hrs-Bar Graph

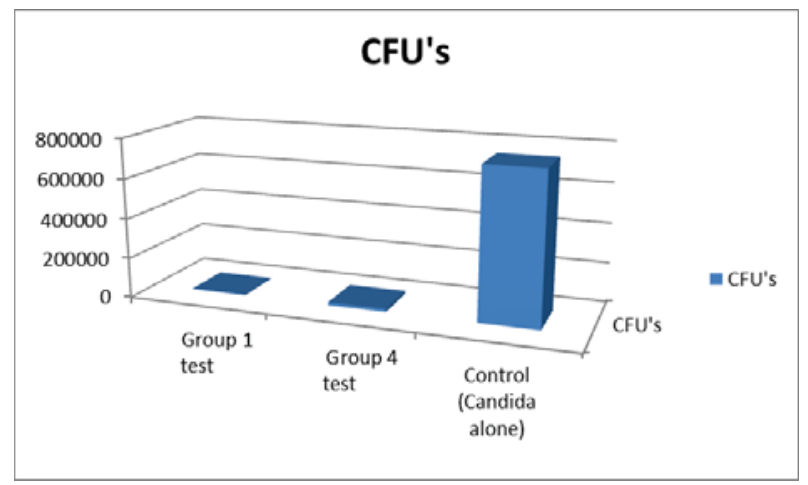

- Table (14). CFU/mL Enterococci faecalis -72hrs

\begin{tabular}{|l|l|l|l|}
\hline Test Organism & $\begin{array}{l}\text { Group 1 test } \\
\text { (E. f and Group 1) }\end{array}$ & $\begin{array}{l}\text { Group 4 test } \\
(\text { E. f and Group 4) }\end{array}$ & $\begin{array}{l}\text { Control(E .faecalis } \\
\text { alone) }\end{array}$ \\
\hline E.Faecalis colonies & $10^{-1}$ & $10^{-1}$ & $1.75 \times 10^{6}$ \\
\hline
\end{tabular}

Figure 61: CFU/ml For Enterococcus faecalis (control), Group I and Group IV mixed with Poloxamer-Bar Graph

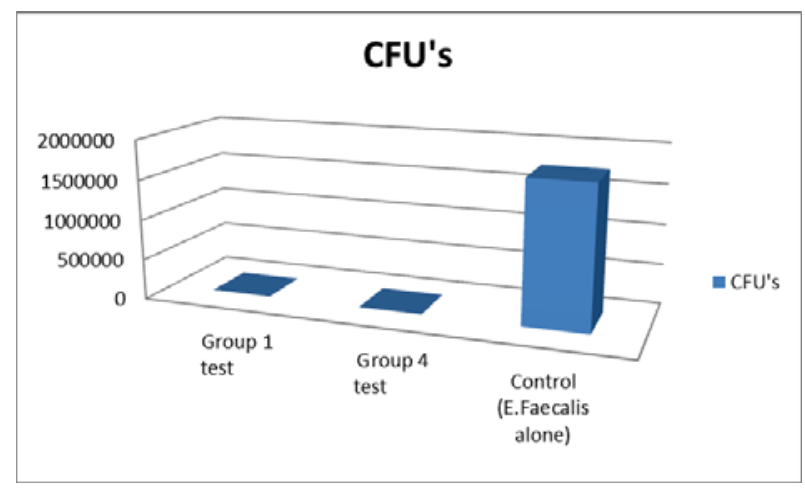




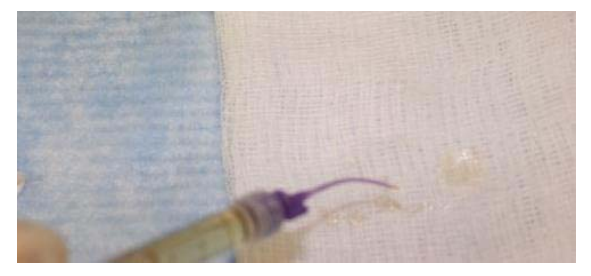

Figure 62: Poloxamer gel with group 1 in a syringe for intra-canal delivery.

\section{$\underline{\text { Discussion }}$}

The main goal of endodontic therapy is to obtain an effective cleaning and decontamination of the root canal system. Traditional endodontic techniques employ mechanical instrumentation, chemical irrigation and irrigant activation devices such as sonics, ultrasonics and lasers, to assist in the attempt to clean, shape and decontaminate all areas of the root canal. Even with all the advancements in technology, endodontic therapy still falls short of successfully removing all of the infective microorganisms and debris. This is due to the complexity of the root canal anatomy and the inability of common irrigants to penetrate into lateral canals and apical ramifications. Therefore, it seems appropriate to search for new materials, techniques and technologies that can improve the cleaning and decontamination of these anatomical areas.

In this study, an innovative approach which might aid in increasing success of endodontic therapy was investigated. This innovative approach involves Bacteriotherapy by allowing probiotic organisms to eliminate pathogenic organisms, either by outcompeting/immunemodulation or by secreting antimicrobial substances such as peroxides. The rationale behind this innovative Bacteriotherapy model evolved after extensive research was done to uncover any missing links in endodontic treatment. Due to the fact that we cannot sterilize a root canal system because of its complex anatomical structure, it was hypothesized that microorganisms existing after treatment and which are considered healthy co-existents at a level where the human body is able to resist damage or destruction to its tissues, might decrease the incidence of endodontic failure. To date, total elimination of bacteria from the root canal system has been the focus of all endodontic procedures, but the fact that some organisms are beneficial throughout the human body has been ignored. That same condition might also exist within root canals of human teeth. In addition to eliminating and out competing the pathogens that 
originally entered from the carious process, probiotic organisms could well not only eliminate disease causing bacteria, but might also prevent their re-establishment after treatment has been completed. It must be understood that the first life forms on earth were bacteria, appearing over 4 billion years ago, and we continue to live in the age of bacteria dominance. All subsequent forms of life evolved to their present states, interacting and integrating with them. Human beings appear to be free of bacteria until they pass through the heavily colonized birth canal, and arrive in the microbial world in which we reside. Our exposed organs and digestive tract become niches for adapted microbes (probiotics). These spaces reflect in part, the exterior environment of our bodies. The contents of these organs are kept separated from the "interior" of the body by barriers that effectively cordon off the luminal microbes. Humans have a developmental process for the expression of antimicrobial peptides which modulate the microbial ecosystem that begins to form at birth. The process of colonization is dynamic, and creates the structured populations reached in the climax community. ${ }^{(28)}$ This aggregate of organisms that resides are found in various areas such as the oral cavity, saliva, conjuctival fluid, skin, gastrointestinal tract, as well as numerous other areas of the body is defined as "The Human Microbiome". ${ }^{27,28)}$

One of the most studied organs which contain microbial communities is the human gastrointestinal tract which contains a very complex and dynamic microbial community, specific for each person depending upon environmental and genetic factors. Each individual holds approximately $1.5 \mathrm{~kg}$ of bacteria within in their gastrointestinal tract. . ${ }^{(12,13,14,17,18,19,27,28)}$

The gut microbiota plays an important role in human health, not only due to its participation in the digestion process, but also for its critical function in the development of the gut and the immune system, along with regulation of absorption and storage of lipids. Recent studies have suggested that specific aberrations in gut microbiota composition are associated with different diseases. The rationale for modulating the gut microbiota by means of probiotics is derived from the importance of gut microbiota on host health. ${ }^{(27)}$

It was suggested in this study that this very same concept be applied to endodontic therapy owing to the fact that the oral cavity is part of the "Oral Human Microbiome", which in turn is part the "Human Microbiome". (27, 28) 
This study involved two phases: phase one (discovery phase) and phase two (application phase). Phase one was a Kirby-Bauer disc diffusion susceptibility test, utilized to determine the sensitivity or resistance of the pathogenic bacteria (E.faecalis or C. albicans) to various probiotic challenges. The pathogenic organisms were grown on blood agar in the presence of test probiotic groups impregnated filter paper discs. The absence of growth around the discs was an indirect measure of the ability of the test probiotic groups to inhibit growth/out compete the pathogenic organism's E. faecalis or C .albicans.

The results of phase one suggested that organisms such as Lactobacillus rhamnosus, Bifidobacterium bifidum, Lactobacillus acidophilus, Lactobacillus casei, Lactobacillus plantarum, Lactobacillus salivarius, Bifidobacterium longum, Streptococcus thermophilus, Lactobacillus bulgaricus, Lactobacillus paracasei, Bifidobacterium lactis and Bifidobacterium breve possess antimicrobial activity against Enterococcus faecalis and Candida albicans. An observation from this study is that groups 1, 4 and 5 all contained common probiotic organisms including L.acidophillus,L.rhamnosus,L.casei and B.Longum, suggesting future focus involve on these organisms for future studies.

Phase two was considered the application portion of the study, suggesting a novel delivery vehicle for probiotics into the root canal system by utilizing 30\% poloxamer 407 (also known as pluronic F-127) mixed with MRS or TSB broth containing the probiotics. Poloxamer has been utilized in biofilm stage testing of microorganisms. Although this secondary purpose was not the main focus of this study, it investigated poloxamer as a delivery mechanism.

Poloxamers are non-ionic polyethylene oxide (PEO) and polypropylene oxide (PPO) copolymers. They are biocompatible and are used in pharmaceutical formulations such as surfactants, emulsifying agents, solubilizing agents, dispersing agents, and in vivo absorbance enhancer 1; therefore they are considered as "functional excipients" because they are essential components, and play an important role in the formulation ${ }^{(42,43, \text { and } 44)}$ of multiple therapeutic agents. 
Two polymers from this class, poloxamer 188 and poloxamer 407, show inverse thermosensitivity. They are soluble in aqueous solutions at low temperatures (mainly $4^{\circ} \mathrm{C}$ ), but

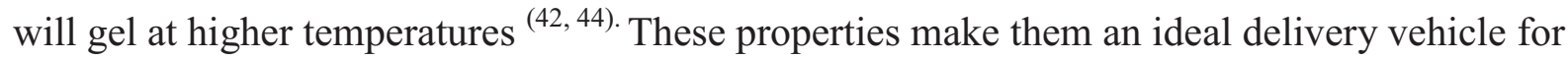
use as an intra-canal medicament between inter-appointment visits.

Other advantages of poloxamer 407, which make it ideal for use in the root canal system is that it has already been employed in topical dosage forms of other surface applied formulations. Poloxamer 407 has been used in vehicles for fluorinated dentifrices, eye applications and contraceptive gels. A poloxamer based dental gel product has been used for treating patients with sensitive gums and teeth. Other properties relevant to this study are that a poloxamer based gel has showed many favorable characteristics for use as a burn dressing by providing a non-toxic detergent covering to the wound. Other studies suggest that the gel itself may accelerate wound healing, making P 407 a suitable vehicle for gels intended to be applied for ulcers and traumatic lesions. It would be an ideal solution for apical periodontitis which is the

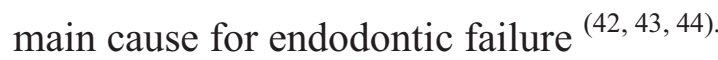

The CFU/ml count results for this study revealed a complete elimination of E. faecalis colony counts for probiotic groups 1 and 4 when mixed in equal amounts with E. faecalis in the $30 \%$ poloxamer/MRS broth formula when compared with controls, and a significant inhibition in $\mathrm{CFU} / \mathrm{ml}$ counts for $C$. albicans in probiotic groups 1 and 4 when mixed in equal amounts with C. albicans in the $30 \%$ poloxamer/MRS broth formula when compared with controls.

The suggested protocol for the use of a poloxamer probiotic based inter-appointment medicament would be to replace the current use of calcium hydroxide. Calcium hydroxide possesses favorable antimicrobial properties, yet it still cannot eliminate all pathogenic organisms such as E. faecalis. The protocol for the probiotic medicament would be, instead of a microbial "elimination therapy" by the use of antimicrobial agents, substituted by a microbial "replacement therapy". Instead of attempting to eliminate pathogenic organisms by antimicrobial agents, probiotics would eliminate the endodontic pathogenic flora, allowing a more favorable/biocompatible environment after standard root canal therapy procedures were followed. This suggested new protocol for therapy would include cleaning, shaping, irrigation and activation of irrigants to decrease the microbial load and remove organic tissue, followed by introducing the poloxamer based probiotic mixture for a period of one week. This would allow 
a more favorable bacterial community to exist by outcompeting or eliminating pathogenic organisms. This would be followed by a second visit of disinfection and obturation to eliminate as many organisms as possible to avoid mutation. If any organisms were to exist after that process, the probiotic "healthy flora" would exist in the root canal system, possibly showing a more favorable endodontic outcome.

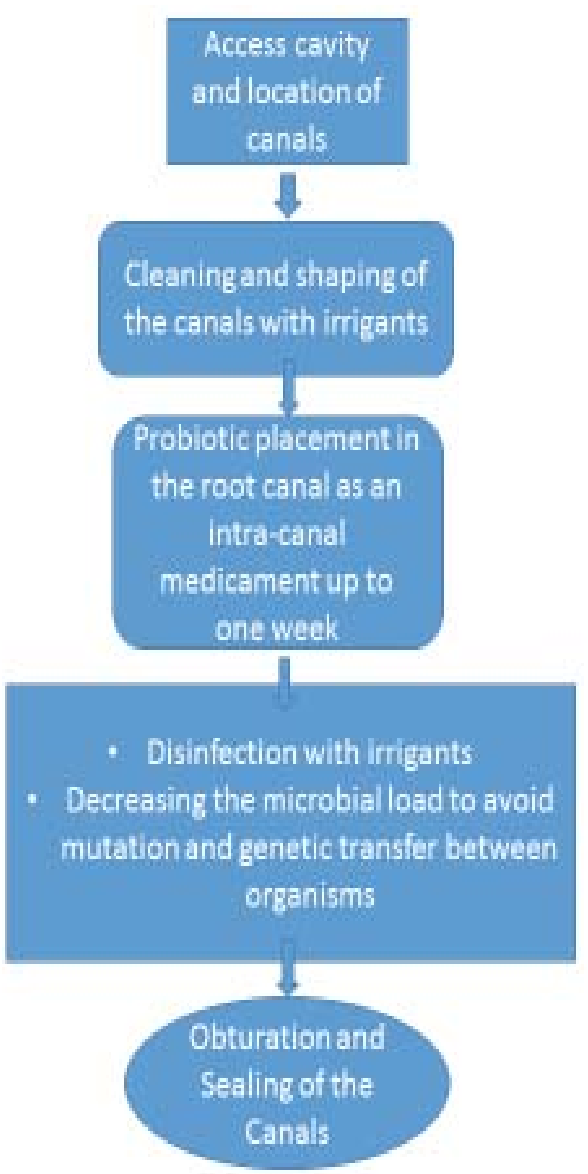




\section{Chapter V}

\section{Conclusion}

The null hypothesis for this study was rejected, since groups I, IV and V showed statistically significant results $(\mathrm{P}<0.05)$ for $\mathrm{ZOI}$ for evaluating planktonic stages, and a reduction in $\mathrm{CFU} / \mathrm{ml}$ counts for biofilm stage testing against $E$. faecalis and $C$. albicans. Within the limitations of this study, it was concluded in phase one that L. acidophilus, L. casei , L. rhamnosus and B. longum were all strains found in common in the probiotic cocktails selected against the endodontic pathogenic organisms E. faecalis and C. albicans in planktonic stages, exhibiting antimicrobial activity which was reflected by the zones of inhibition (ZOI) seen. In phase two it was concluded that probiotic groups I and IV demonstrated a decrease in CFU/ml count of the pathogenic organisms E. faecalis and C. albicans in the biofilm stage when mixed together with the probiotics. Another conclusion is that poloxamer 407 may be used as a delivery vehicle for probiotics in the root canal system as an inter-appointment medicament. This is due to its unique reverse gel physical properties as well as its biocompatibility. This pilot study demonstrated that probiotics show a potential in root canal therapy, but further in vitro and in vivo studies are needed to determine the full potential of "Bacteriotherapy" and its application in endodontics. This model, if successful, may well be introduced and adapted in wound care management, since apical periodontitis can be considered a chronically existing wound, similar in nature to soft tissue wounds that are recalcitrant to standard antimicrobial therapies. 


\section{$\underline{\text { References }}$}

\section{Endodontic Microbiology:}

1. Nair, P. N. R. (2004). Pathogenesis of apical periodontitis and the causes of endodontic failures. Critical Reviews in Oral Biology \& Medicine, 15(6), 348-381. doi:10.1177/154411130401500604

2. KAKEHASHI, S., STANLEY, H. R., \& FITZGERALD, R. J. (1965). The effects of surgical exposures of dental pulps in germ-free and conventional laboratory rats. Oral Surgery, Oral Medicine, and Oral Pathology, 20, 340-349.

3. Sundqvist, G. (1994). Taxonomy, ecology, and pathogenicity of the root canal flora. Oral Surgery, Oral Medicine, and Oral Pathology, 78(4), 522-530.

4. Gomes, B. P., Pinheiro, E. T., Gade-Neto, C. R., Sousa, E. L., Ferraz, C. C., Zaia, A. A .Souza-Filho, F. J. (2004). Microbiological examination of infected dental root canals. Oral Microbiology and Immunology, 19(2), 71-76.

5. Chavez de Paz, L. (2004). Gram-positive organisms in endodontic infections. Endodontic Topics, 9(1), 79-96.

6. Fabricius, L., Dahlen, G., Holm, S. E., \& Moller, A. J. (1982). Influence of combinations of oral bacteria on periapical tissues of monkeys. Scandinavian Journal of Dental Research, 90(3), 200-206.

7. Fabricius, L., Dahlen, G., Sundqvist, G., Happonen, R. P., \& Moller, A. J. (2006). Influence of residual bacteria on periapical tissue healing after chemomechanical treatment and root filling of experimentally infected monkey teeth. European Journal of Oral Sciences, 114(4), 278-285. doi:10.1111/j.1600-0722.2006.00380.x 
8. Fujii, R., Saito, Y., Tokura, Y., Nakagawa, K. Okuda, K., \& Ishihara, K. (2009). Characterization of bacterial flora in persistent apical periodontitis lesions. Oral Microbiology and Immunology, 24(6), 502-505. doi:10.1111/j.1399-302X.2009.00534.

9. Seltzer, S., \& Bender, I. B. (2003). Cognitive dissonance in endodontics. 1965. Journal of Endodontics, 29(11), 714-9; discussion 713.

\section{Probiotics:}

10. Al-Dajani, M., \& Limeback, H. (2012). Emerging science in the dietary control and prevention of dental caries. Journal of the California Dental Association, 40(10), 799-804.

11. Allaker, R. P., \& Douglas, C. W. (2009). Novel anti-microbial therapies for dental plaquerelated diseases. International Journal of Antimicrobial Agents, 33(1), 8-13. doi:10.1016/j.ijantimicag.2008.07.014; 10.1016/j.ijantimicag.2008.07.014

12. Borchers, A. T., Selmi, C., Meyers, F. J., Keen, C. L., \& Gershwin, M. E. (2009). Probiotics and immunity. Journal of Gastroenterology, 44(1), 26-46. doi:10.1007/s00535008-2296-0; 10.1007/s00535-008-2296-0

13. Elavarasu, S., Jayapalan, P., \& Murugan, T. (2012). Bugs that debugs: Probiotics. Journal of Pharmacy \& Bioallied Sciences, 4(Suppl 2), S319-22. doi:10.4103/0975-7406.100286; $10.4103 / 0975-7406.100286$

14. Grover, H. S., \& Luthra, S. (2012). Probiotics-the nano soldiers of oral health. Journal, Indian Academy of Clinical Medicine, 13(1), 49.

15. Harini, P. M., \& Anegundi, R. T. (2010). Efficacy of a probiotic and chlorhexidine mouth rinses: A short-term clinical study. Journal of the Indian Society of Pedodontics and 
Preventive Dentistry, 28(3), 179-182. doi:10.4103/0970-4388.73799; 10.4103/09704388.73799

16. Khanafari, A., Porgham, S. H., \& Ebrahimi, M. T. (2012). Investigation of probiotic chocolate effect on streptococcus mutans growth inhibition. Jundishapur Journal of Microbiology, 5(4), 590-597. doi:10.5812/jjm.3861

17. Meurman, J. H. (2005). Probiotics: Do they have a role in oral medicine and dentistry? European Journal of Oral Sciences, 113(3), 188-196. doi:10.1111/j.16000722.2005.00191.x

18. Reddy, J. J., Sampathkumar, N., \& Aradhya, S. (2010). Probiotics in dentistry: Review of the current status; probiótica em odontologia: Revisão do estado atual da questão. Rev.Clín.Pesq.Odontol.(Impr.), 6(3), 261-267.

19. Reddy, S., Swapna, L., Ramesh, T., Singh, R., Vijayalaxmi, N., \& Lavanya, R. (2011). Bacteria in oral health-probiotics and prebiotics a review.

20. Salminen, S., von Wright, A., Morelli, L., Marteau, P., Brassart, D., de Vos, W. M., Mattila-Sandholm, T. (1998). Demonstration of safety of probiotics -- a review. International Journal of Food Microbiology, 44(1-2), 93-106.

21. Slawik, S., Staufenbiel, I., Schilke, R., Nicksch, S., Weinspach, K., Stiesch, M., \& Eberhard, J. (2011). Probiotics affect the clinical inflammatory parameters of experimental gingivitis in humans. ( No. 65). doi:10.1038/ejen.2011.45

22. Twetman, S. (2012). Are we ready for caries prevention through bacteriotherapy? Brazilian Oral Research, 26 Suppl 1, 64-70.

23. Gill, H. S., \& Guarner, F. (2004). Probiotics and human health: A clinical perspective. Postgraduate Medical Journal, 80(947), 516-526. doi:10.1136/pgmj.2003.008664 
24. Krasse, P., Carlsson, B., Dahl, C., Paulsson, A., Nilsson, A., \& Sinkiewicz, G. (2006). Decreased gum bleeding and reduced gingivitis by the probiotic lactobacillus reuteri. Swedish Dental Journal, $30(2), 55-60$.

25. Haukioja, A. (2010). Probiotics and oral health. European Journal of Dentistry, 4(3), 348.

26. Caglar, E., Kargul, B., \& Tanboga, I. (2005). Bacteriotherapy and probiotics' role on oral health. Oral diseases, 11(3), 131-137.

27. Gueimonde, M., \& Collado, M. D. C. (2012). Metagenomics and probiotics.Clinical Microbiology and Infection, 18(s4), 32-34.

28. Dominguez-Bello, M. G., \& Blaser, M. J. (2008). Do you have a probiotic in your future?. Microbes and Infection, 10(9), 1072-1076.

29. Roberfroid, M. (2007). Prebiotics: the concept revisited. The Journal of nutrition,137(3), 830S-837S.

30. Surawicz, C. M., Elmer, G. W., Speelman, P. I. E. T. E. R., McFarland, L. V., Chinn, J., \& Van Belle, G. (1989). Prevention of antibiotic-associated diarrhea by Saccharomyces boulardii: A prospective study. Gastroenterology, 96(4), 981-988.

31. Kragen $\mathrm{H}$. The treatment of inflammatory affections of the oral mucosa with a lactic acid bacterial culture preparation. Zahnarztl Welt 1954;9:306-308.

32. Krasse P, Carlsson B, Dahl C, Paulsson A, Nilsson A, Sinkiewicz G. Decreased gum bleeding and reduced gingivitis by the probiotic Lactobacillus reuteri. Swed Dent J 2006;30:55-60.

33. Della Riccia DN, Bizzini F, Perilli MG, Polimeni A, Trinchieri V, Amicosante G, et al. Anti-inflammatory effects of Lactobacillus brevis (CD2) on periodontal disease. Oral Dis 2007;13:376-385. 
34. Twetman S, Derawi B, Keller M, Ekstrand K, Yucel-Lindberg T, Stecksen-Blicks C. Short-term effect of chewing gums containing probiotic Lactobacillus reuteri on the levels of inflammatory mediators in gingival crevicular fluid. Acta Odontol Scand 2009;67:1924.

35. Ahola AJ, Yli-Knuuttila H, Suomalainen T, Poussa T, Ahlstrom A, Meurman JH, et al. Short-term consumption of probiotic-containing cheese and its effect on dental caries risk factors. Arch Oral Biol 2002;47:799-804.

36. Delanghe G, Ghyselen J, van Steenberghe D, Feenstra L. Multidisciplinary breath-odour clinic. The Lancet 1997;350:187-187.

37. Evans, M., Davies, J. K., Sundqvist, G., \& Figdor, D. (2002). Mechanisms involved in the resistance of Enterococcus faecalis to calcium hydroxide. International Endodontic Journal, 35(3), 221-228.

38. Stuart, C. H., Schwartz, S. A., Beeson, T. J., \& Owatz, C. B. (2006). Enterococcus faecalis; Its Role in Root Canal Treatment Failure and Current Concepts in Retreatment. Journal of endodontics, 32(2), 93-98.

39. Costerton, J. W., Lewandowski, Z., Caldwell, D. E., Korber, D. R., \& Lappin-Scott, H. M. (1995). Microbial biofilms. Annual Reviews in Microbiology, 49(1), 711-745.

40. Svensäter, G., \& Bergenholtz, G. (2004). Biofilms in endodontic infections. Endodontic topics, 9(1), 27-36.

41. Ramage, G., Mowat, E., Jones, B., Williams, C., \& Lopez-Ribot, J. (2009). Our current understanding of fungal biofilms. Critical reviews in microbiology, 35(4), 340-355. 


\section{Poloxamer:}

42. Patel, H. R., Patel, R. P., \& Patel, M. M. (2009). Poloxamers: A pharmaceutical excipients with therapeutic behaviors. International Journal of PharmTech Research, 1(2).

43. Cafaggi, S., Russo, E., Caviglioli, G., Parodi, B., Stefani, R., Sillo, G., ... \& Bignardi, G. (2008). Poloxamer 407 as a solubilising agent for tolfenamic acid and as a base for a gel formulation. european journal of pharmaceutical sciences, 35(1), 19-29.

44. Nalbandian, R. M., Henry, R. L., Balko, K. W., Adams, D. V., \& Neuman, N. R. (1987). Pluronic F-127 gel preparation as an artificial skin in the treatment of third-degree burns in pigs. Journal of biomedical materials research, 21(9), 1135-1148.

45. Friedman, S., Abitbol, S., \& Lawrence, H. P. (2003). Treatment outcome in endodontics: the Toronto Study. Phase 1: initial treatment. Journal of Endodontics, 29(12), 787-793. 


\section{ABSTARCTS AND PRESENTATIONS}

- Maximizing Clinical Benefits of Probiotics: Matching Metagenomics, Patient age and Microbial CompositionOralHealthGroup-December2013

- Salvation of Endodontics: Anti-Biofilm Medicament using Probiotics (Part 1 Discovery)-Van Liere WVU-2014

- Endotracheal Biofilm Architecture by SEM: Dental Plaque Origin for Pneumonia-AADR \IADR 2014

- Probiotics: The Nano Army of Endodontics.-AAE 2014 